\title{
The numerical analysis of the effect of Grashof number, modified Grashof number and chemical reaction on the non-Darcy MHD flow of a Casson fluid over a nonlinearly stretching sheet in a porous medium
}

\author{
Bhim Sen Kala \\ Doon University, Dehradun,248001,Uttarakhand, India \\ Corresponding author: bhimskala@gmail.com.
}

Available online at: www.isroset.org

Received: 11/May/2019, Accepted: 02/Jun/2019, Online: 30/Jun/2019

\begin{abstract}
In this paper, we have studied the numerical analysis of the effect of Grashof number, modified Grashof number and chemical reaction on the non-Darcy MHD flow of a Casson fluid over a nonlinearly stretching sheet in a porous medium. In the mathematical model, using similarity variables, the momentum, energy and concentration equations are transformed to non-dimensional ordinary differential equations.. And then these are solved numerically using bvp4c method, a Matlab inbuilt bvp4c-programm. A discussion for the effects of the parameters involved on the boundary layer regions and the magnitude of the velocity, temperature and concentration and Local skin friction, Local Nusselt Number and Local Sherwood Number have been done graphically and numerically using figures and tables.
\end{abstract}

Keywords- Casson fluid; magnetic parameter; darcy parameter; velocity slip parameter; forchheimrer parameter; power index parameter.inclination Grashof number, modified Grashof number.

Mathematics Subject Classification: 35A22, 35A35, 35A99, 35G20, 35G30

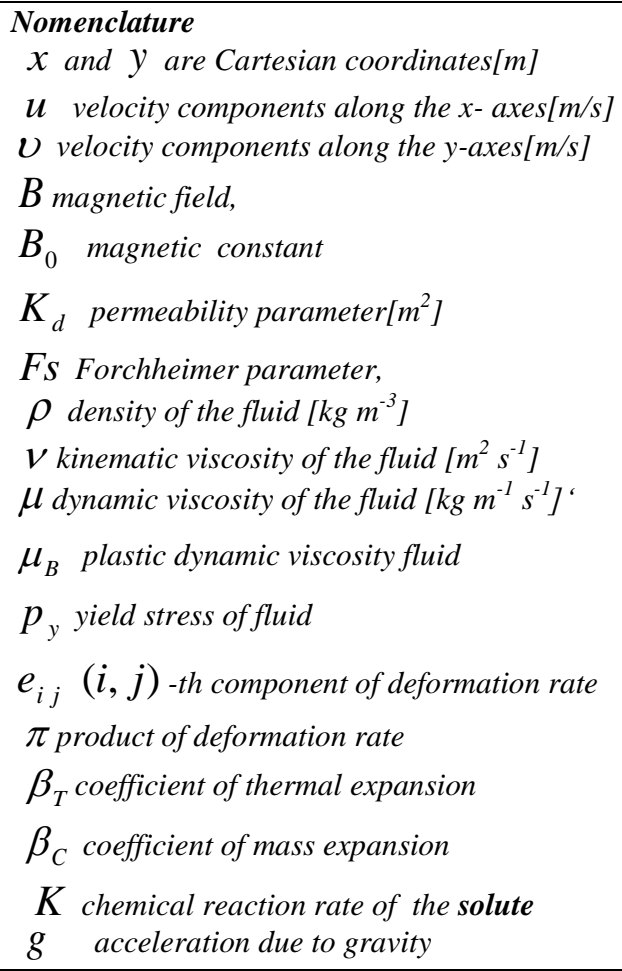

$K_{p}$ non-dimensional Permeability parameter

$C_{b}$ non-dimensional Forchheimer coefficient

Gr Grashof number,

Gc solutal Grashof number,

$\gamma^{*}$ buoyancy parameter,

$\gamma$ solutal buoyancy parameter,

$K_{c}$ chemical reaction parameter

$u_{S}$ velocity slip

$U_{w}$ wall mass transfer velocity

VS Velocity slip parameter

$n_{\text {stretching surface power index parameter. }}$ temperature at the surface

$T_{w}$ initial temperature of the surface

$T_{0} \quad$ proportionality constant

$\frac{\partial T}{\partial y}$ temperature gradient term 


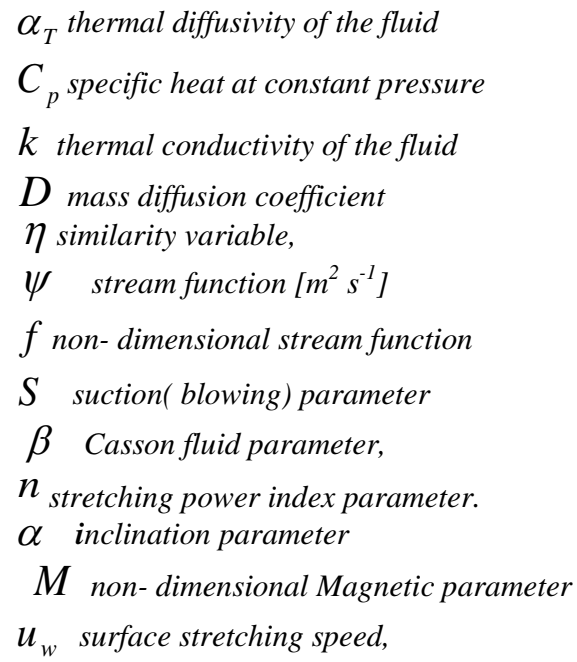

$C(x)$ concentration at the surface

$C_{w}$ initial concentration of the surface

$C_{0}$ proportionality constant

$\frac{\partial C}{\partial y}$ concentration gradient term

$T_{\infty}$ ambient temperature

$C_{\infty}$ ambient concentration

$C_{f}$ local skin-friction

$\operatorname{Re}_{f}$ Reynold number.

$\tau_{w}$ Shearing stress

$\mathrm{Nu}_{x} \quad$ local Nusselt number

Sh ${ }_{x}$ local Sherwood number

\section{INTRODUCTION}

Fluid flow over a stretching sheet has many important applications: in polymer sheet manufacturing, in chemical engineering, and in metal processing in metallurgy etc. Crane [1] first initiated the study of flow of Newtonian viscous incompressible fluid over a linearly stretching sheet. $\mathrm{He}$ investigated the flow of viscous incompressible fluid along a stretching plate whose velocity is proportional to the distance from the slit; such situation occurs in drawing of plastic films. The study was extended to non-Newtonian fluids by many researchers. Rajagopal [2] studied the flow of viscous incompressible fluid on moving (stretching) surface in the boundary layer region. Ishak et al. [3] investigated the MHD flow of viscous incompressible fluid along a moving wedge under the condition of suction and injection.

Non -Newtonian types of flow occurs in the drawing of plastic films and artificial fibres. The moving fibre produces a boundary layer in the medium. Surrounding medium of the fibre is of technical importance; in that it governs the rate at which the fibre is cooled and this in turn affects the final properties of the yarn. Some of the studies on non-Newtonian fluid are as follows.

Siddappa et al. [4] investigated the flow of visco-elastic fluid (a non-Newtonian fluid) of 'Walters's liquid B Model' for the boundary layer flow past a stretching plate. Andersson [5] investigated the flow of viscoelastic fluid along a stretching sheet in the presence of transverse magnetic field. Dandapat [6] investigated the effect of transverse magnetic field on the stability of flow of viscoelastic fluid over a stretching sheet.
Fang [7] studied that variable transformation method can be used to get the solution of extended Blasius equation from original Blasius equation. Mamaloukas et al. [8] have discussed some alike nature of free-parameter method and separation of variable method and have found exact solution of equation representing flow of two-dimensional viscoelastic second grade fluid over a stretching sheet. Khidir [9] used spectral homotopy perturbation method and successive linearization method to solve Falker-Skan equation (A nonlinear boundary value problem).

Bataller [10] investigated the flow in the boundary layer of the viscous incompressible fluid under two situations: one about a moving plate in a quiescent ambient fluid (Sakiadis flow) and another uniform free stream flow over a resting flat-plate (Blasius flow).

Motsa, et al. [11] investigated the MHD boundary layer flow of upper-convected Maxwell (UCM) fluid over a porous stretching surface. Motsa et al. [12] had analysed the MHD flow of viscous incompressible fluid over a nonlinearly stretching sheet. Rosca [13] discussed the flow of viscous electrically conducting fluid over a shrinking surface in the presence of transverse magnetic field.

Nadeem et al. [14] investigated the MHD boundary layer flow of Williamson fluid over a stretching sheet. Mukhopadhyay [15] analysed the axis symmetric boundary layer flow of viscous incompressible fluid along a stretching cylinder in the presence of uniform magnetic field and under partial slip conditions. Akbar et al. [16] investigate the MHD boundary layer flow of Carreau fluid over a permeable shrinking sheet. 
Nadeem et al. [17] investigated the MHD boundary layer flow of a Casson fluid over an exponentially shrinking sheet. Biswas et al. [18] studied the effects of radiation and chemical reaction on MHD unsteady heat and mass transfer of Casson fluid flow past a vertical plate. Ahmmed et al. [19] analysed the unsteady MHD free convection flow of nanofluid through an exponentially accelerated inclined plate embedded in a porous medium with variable thermal conductivity in the presence of radiation. Biswas et al. [20] investigated the effects of Hall current and chemical reaction on MHD unsteady heat and mass transfer of Casson nanofluid flow through a vertical plate.

Noor et al. [21] investigated the hall current and thermophoresis effects on MHD mixed convective heat and mass transfer thin film flow. Sharada et al. [22] studied MHD mixed convection flow of a Casson fluid over an exponentially stretching surface with the effects of soret, dufour, thermal radiation and chemical reaction. Mukhopadhyay et al. [23] investigated exact solutions for the flow of Casson fluid over a stretching surface with transpiration and heat transfer effects. Pandya et al.[24] have discussed combined effect of variable permeability and variable magnetic field on mhd flow past an inclined plate with exponential temperature and mass diffusion with chemical reaction through porous media.

Kala [25] studied the analysis of non-Darcy MHD flow of a Casson fluid over a non-linearly stretching sheet with partial slip in a porous medium.

This work is the extension of the work [25] in which analysis of non-Darcy MHD flow of a Casson fluid over a non-linearly stretching sheet with partial slip in a porous medium is studied.

This work deals with the numerical analysis of the effect of Grashof number, modified Grashof number and chemical reaction on the non-Darcy MHD flow of a Casson fluid over a nonlinearly stretching sheet in a porous medium.

\section{MATHEMATICAL MODELLING}

We consider steady two-dimensional laminar boundary-layer flow of viscous, incompressible, electrically conducting nonNewtonian Casson fluid in a saturated homogeneous nonDarcy porous medium caused by nonlinearly stretching sheet, which is inclined with an acute angle $(\alpha)$ to the vertical, placed at the bottom of the porous medium. The xaxis is taken along the stretching surface in the direction of the motion while the $y$-axis is normal to the surface. A Cartesian coordinate system is used. The $x$-axis is along the direction of the continuous stretching surface (the sheet) and $y$-axis is normal to the $x$-axis. The sheet is assumed to be stretched along the $x$-axis, keeping the position of the origin unaltered and stretching velocity varies nonlinearly with the distance from the origin. A uniform magnetic field of strength $B$ is applied normal to the sheet.

It is assumed that the fluid is optically dense, nonNewtonian, and without phase change. Flow region is in non-Darcy porous medium. This integrates a linear Darcian drag for low velocity effects (bulk impedance of the porous matrix at low Reynolds numbers) and a quadratic (second order) resistance, the Forchheimer drag force, for high velocity flows, as may be come across in chemical engineering systems operating at higher velocities. Brinkman's equation takes into account the boundary effects (the viscous force).

It is assumed that the induced magnetic field, the external electric field and the electric field due to polarization of charges are negligible in comparison to the applied magnetic field. So, all of the Hall effects and Joule heating effects are neglected.

The viscosity and thermal conductivity of the fluid are assumed to be constant. The temperature and concentration of the stretching surface are always greater than their free stream values.

Under these assumptions the rheological equation for incompressible flow of Casson fluid is given by (Sharada et al. [22], Mukhopadhyay et al. [23])

$$
\begin{gathered}
\tau_{i j}=2 e_{i j}\left(\mu_{B}+\frac{p_{y}}{\sqrt{2 \pi}}\right) \text { if } \pi>\pi_{c} \\
\tau_{i j}=2 e_{i j}\left(\mu_{B}+\frac{p_{y}}{\sqrt{2 \pi_{c}}}\right) \text { if } \pi<\pi_{c}
\end{gathered}
$$

Where $\mu$ is the dynamic viscosity, $\mu_{B}$ is plastic dynamic viscosity of the non-Newtonian fluid, $p_{y}$ is the yield stress of fluid, $\pi$ is the product of the component of deformation rate with itself, namely, $\pi=e_{i j} e_{i j} \quad$ and $e_{i j}$ is the $(i, j)$-th component of the deformation rate. $\pi_{c}$ is critical value of the product based on the non- Newtonian model. The flow configuration and the coordinate system are shown in Figure 1.

Under these assumptions, the governing boundary layer equations for momentum, energy and mass take the following form: 


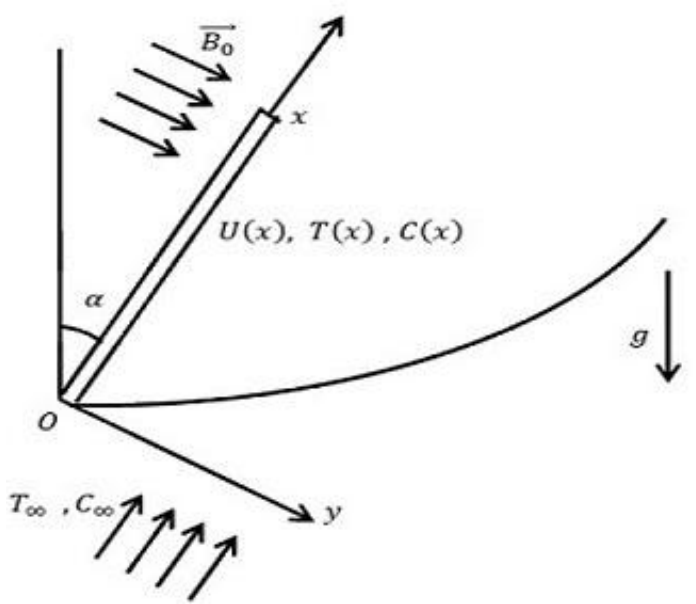

Figure 1 Physical model and coordinate system (for stretching sheet)

The equation of continuity:

$\frac{\partial u}{\partial x}+\frac{\partial v}{\partial y}=0$

The Equation of Momentum:

$u \frac{\partial u}{\partial x}+v \frac{\partial u}{\partial y}=\left(1+\frac{1}{\beta}\right) \frac{\partial^{2} u}{\partial y^{2}}$

$\pm g\left(\beta_{T}\left(T-T_{\infty}\right)+\beta_{C}\left(C-C_{\infty}\right)\right) \cos \alpha$

$-\frac{\sigma B_{0}^{2}}{\rho_{f}} u-\frac{v}{K_{d}} u^{\prime}-\frac{C_{b}}{\sqrt{K_{d}}} u^{2}$

The Equation of Energy:

$u \frac{\partial T}{\partial x}+v \frac{\partial T}{\partial y}=\alpha \frac{\partial^{2} T}{\partial y^{2}}$

The Equation of Mass concentration:

$$
\begin{aligned}
& u \frac{\partial C}{\partial x}+v \frac{\partial C}{\partial y}=D \frac{\partial^{2} C}{\partial y^{2}} \\
& -K_{c}\left(C-C_{\infty}\right)
\end{aligned}
$$

where, the sign \pm refers to the cases of assisting and opposing flow (here we shall consider the case of assisting flow which is shown by positive sign), $x$ and $y$ are cartesian coordinates along the stretching sheet and normal to it respectively, $u$ and $v$ are the velocity components along the $x$-and $y$-axes, $K_{d}$ is the permeability of the porous medium, $B$ is magnetic field, $C_{b}$ is Forchheimer coefficient, $\alpha$ is angle of inclination of the surface with the vertical, $\beta$ is the Casson fluid parameter, $\rho, v$ and $\mu$ are density, kinematic viscosity and dynamic viscosity of the fluid respectively. $\beta_{T}, \beta_{C}$ and $K$ are the coefficient of thermal expansion, the coefficient of mass expansion and the chemical reaction rate of the solute, respectively, $g$ is acceleration due to gravity, $\alpha_{T}=\frac{k}{\rho C_{p}} \alpha=k /\left(\rho C_{p}\right)$ is the thermal diffusivity of the fluid $C_{p}$ is the specific heat at constant pressure, $k$ is the thermal conductivity of the fluid, and $D$ is mass diffusion coefficient.

The strength of the magnetic field is assumed to vary spatially by $B(x)=B_{0} x^{(n-1) / 2}$ where $B_{0}$ is constant.

The sheet is assumed to move with power law velocity, and varies nonlinearly in spatial coordinates with some index, in the boundary layer region, so that relevant velocity boundary conditions for equations (1) to (4) are as follows:

$$
\begin{aligned}
& \text { At } y=0: u=U(x)=u_{w}+u_{w} \\
& =c x^{n}+N \mu \frac{\partial u}{\partial y}, \\
& v=v_{w}=-V(x),-\infty<x<\infty \\
& T(x)=T_{w}+T_{0} \frac{\partial T}{\partial y}, \\
& C(x)=C_{w}+C_{0} \frac{\partial C}{\partial y} ; \\
& A s \quad y \rightarrow \infty: u \rightarrow 0, \\
& T \rightarrow T_{\infty}, C \rightarrow C_{\infty} .
\end{aligned}
$$

where $u_{w}$ is the surface velocity of the sheet with $u_{w}=c x^{n}$ where $c$ is a constant parameter related to the surface stretching speed, $u_{S}$ is the velocity slip which is assumed to be proportional to the local shear stress as follows: $u_{S}=N \mu \frac{\partial u}{\partial y}=N\left(\mu_{B}+p_{y} / \sqrt{2 \pi}\right) \frac{\partial u}{\partial y}$, where $N$ $\left(m^{2} s \mathrm{~kg}^{-1}\right)$ is the slip constant. $\mu=\left(\mu_{B}+p_{y} / \sqrt{2 \pi}\right)$ is dynamic viscosity. $\beta=\mu_{B} \sqrt{2 \pi} / p_{y}$ is Casson fluid parameter. $v_{w}$ is the wall mass transfer velocity with $v_{w}>0$ for mass suction and $v_{w}<0$ for mass injection. $n$ 
is the stretching surface index parameter, $n>0$ is for accelerated sheet and $n<0$ is for decelerated sheet.

$T(x)$ is temperature at the surface at a distance $x$ along the surface from the origin, $T_{W}$ is initial temperature of the surface, $T_{0}$ is proportionality constant arising in dealing with temperature gradient term $\frac{\partial T}{\partial y} \cdot C(x)$ is concentration at the surface at a distance $x$ along the surface from the origin, $C_{W}$ is initial concentration of the surface, $C_{0}$ is proportionality constant arising in dealing with concentration gradient term $\frac{\partial C}{\partial y} . T_{\infty}$ and $C_{\infty}$ are ambient temperature and concentration respectively.

Dimensional analysis: We define

$$
\begin{aligned}
& \eta=\left(\frac{c(n+1)}{2 v}\right)^{\frac{1}{2}} x^{\frac{n-1}{2}} y, \\
& \psi=\left(\frac{2 c v}{(n+1)}\right)^{\frac{1}{2}} x^{\frac{n+1}{2}} f(\eta), \\
& u=\frac{\partial \psi}{\partial y}, v=-\frac{\partial \psi}{\partial x}, \\
& u=c x^{n} f^{\prime}(\eta) \\
& v=-\left(\frac{c v(n+1)}{2}\right)^{\frac{1}{2}} x^{\frac{n-1}{2}} \\
& \left(f(\eta)+\frac{(n-1)}{(n+1)} \eta f^{\prime}(\eta)\right) \\
& \theta(\eta)=\frac{\left(T-T_{\infty}\right)}{\left(T_{w}-T_{\infty}\right)}, \\
& \phi(\eta)=\frac{\left(C-C_{\infty}\right)}{\left(C_{w}-C_{\infty}\right)}
\end{aligned}
$$

Here $\eta$ is similarity variable, $\psi$ is stream function, $f$ is non-dimensional stream function, $u$ is $x$-component of velocity, $v$ is $y$ - component of velocity.

Using equations (6), equations (1) to (4) can be written as

$$
\begin{gathered}
\left(1+\frac{1}{\beta}\right) f^{\prime \prime \prime}+f f^{\prime \prime}-\frac{2 n}{n+1} f^{\prime 2} \\
+\frac{2}{n+1}\left(\gamma^{*} \theta+\gamma \phi\right) \cos \alpha \\
-\frac{2}{n+1}\left(\begin{array}{l}
\left.\left(M+\left(1 / K_{p}\right)\right) f^{\prime}\right) \\
+F s\left(f^{\prime}\right)^{2}
\end{array}\right)=0 \\
\frac{1}{\operatorname{Pr}} \theta^{\prime \prime}+f \theta^{\prime}=0 \\
\frac{1}{S c} \phi^{\prime \prime}+f \phi^{\prime} \\
-\frac{2}{n+1} K_{c} \phi=0
\end{gathered}
$$

And boundary conditions (5) as ,

$$
\begin{aligned}
& f(0)=S, \\
& f^{\prime}(0)=1+V s f^{\prime \prime}(0), \\
& \theta(0)=1+\lambda t \theta^{\prime}(0), \\
& \phi(0)=1+\lambda c \phi^{\prime}(0) \\
& f^{\prime}(\eta) \rightarrow 0, \theta(\eta) \rightarrow 0, \\
& \phi(\eta) \rightarrow 0 \text { as } \eta \rightarrow \infty
\end{aligned}
$$

where

$$
\begin{aligned}
& \lambda t=T_{0}\left(\frac{c(n+1)}{2 v}\right)^{\frac{1}{2}} x^{\frac{(n-1)}{2}}, \\
& \lambda c=C_{0}\left(\frac{c(n+1)}{2 v}\right)^{\frac{1}{2}} x^{\frac{(n-1)}{2}}
\end{aligned}
$$

Here prime denotes differentiation with respect to $\eta$.

The parameters occurring in equations (7) to (10) are defined as follows: 
$M=\frac{\sigma B_{0}^{2}}{c \rho_{f}}$ is Magnetic parameter $K_{p}=\frac{c K_{d} x^{(n-1)}}{v}$

is Permeability parameter, $F s=\frac{C_{b} x}{\sqrt{K_{d}}} \quad F s=\frac{C_{b} x}{\sqrt{K_{d}}}$ is

Forchhiemer parameter, $G r=\frac{g \beta_{T}\left(T_{w}-T_{\infty}\right) x^{2}}{v^{2}} \quad$ is Grashof number, $G c=\frac{g \beta_{C}\left(C_{w}-C_{\infty}\right) x^{2}}{v^{2}}$ is solutal Grashof number, $\gamma^{*}=\frac{G r}{\mathrm{Re}^{2}}$ is buoyancy parameter, $\gamma=\frac{G c}{\operatorname{Re}^{2}}$ is solutal buoyancy parameter, $\operatorname{Re}_{x}=\frac{u_{w} x}{v}$ is Reynolds number, $u_{w}=c x^{n}$ fluid velocity along the wall surface, $\quad S c=\frac{v}{D}$ is Schmidt number, $\operatorname{Pr}=\frac{v}{\alpha_{T}} \quad$ is Prandtl number, $K_{c}=K^{-1} x^{1-n}$ is reaction rate parameter, $K_{c}>0$ represents destructive chemical reaction, $K_{c}=0$ corresponds to no reaction and $K_{c}<0$, stands for generative chemical reaction. $S=-v_{w} \sqrt{\frac{2}{v c(n+1)}} x^{\frac{(1-n)}{2}} \quad$ is suction/injection parameter, $S>0$ for suction and $S<0$ for injection or blowing $\left(v_{w}<0\right.$ for mass suction and $v_{w}>0$ for mass injection). $V s=N \mu \sqrt{\frac{c(n+1)}{2 v}} x^{\frac{(n-1)}{2}}$ is velocity slip parameter. $\lambda t$ is thermal slip parameter. $\lambda c$ is concentration slip parameter.

The engineering design quantities which have physical interest include local Skin-friction coefficient $C f_{x}$ local Nusselt number $N u_{x}$ and local Sherwood number $S h_{x}$ and Reynold number $\left(R e_{x}\right)$, are given as follows:

The local Skin-friction coefficient is $\quad C f_{x}=\frac{\tau_{w}}{\rho_{f} u_{w}^{2}}$

The Shearing Stress is $\tau_{w}=\mu_{B}\left(1+\frac{1}{\beta}\right)\left(\frac{\partial u}{\partial y}\right)_{y=0}$,
$\tau_{W}=\mu\left(\frac{\partial u}{\partial y}\right)_{y=0}=\left(\mu_{B}+\frac{p_{y}}{\sqrt{2 \pi}}\right)\left(\frac{\partial u}{\partial y}\right)_{y=0}$

The local Reynold number is $\operatorname{Re}_{x}=\frac{u_{w} x}{v}$,

$$
\begin{aligned}
& C f_{x}=\frac{\left(\mu_{B}+\frac{p_{y}}{\sqrt{2 \pi}}\right)\left(\frac{\partial u}{\partial y}\right)_{y=0}}{\rho_{f} u_{w}^{2}} \\
& =\frac{1}{\sqrt{\operatorname{Re}_{x}}} \sqrt{\frac{n+1}{2}}\left(1+\frac{1}{\beta}\right) f^{\prime \prime}(0)
\end{aligned}
$$

Local Nusselt number

$$
\begin{aligned}
& N u_{x}=\frac{-x\left(\frac{\partial T}{\partial y}\right)_{y=0}}{T_{w}-T_{\infty}} \\
& =-\sqrt{\operatorname{Re}_{x}} \sqrt{\frac{n+1}{2}} \theta^{\prime}(0)
\end{aligned}
$$

Local Sherwood number:

$$
\begin{gathered}
S h_{x}=\frac{-x\left(\frac{\partial C}{\partial y}\right)_{y=0}}{T_{w}-T_{\infty}} \\
=-\sqrt{\operatorname{Re}_{x}} \sqrt{\frac{n+1}{2}} \phi^{\prime}(0)
\end{gathered}
$$

\section{METHOD OF NUMERICAL SOLUTION}

The numerical solutions are obtained using the above equations for some values of the governing parameters, namely, the Magnetic parameter $(M)$, the Permiability porosity parameter $(K p)$, the Forchhemier parameter $(F s)$, inclination $\operatorname{parameter}(\alpha)$, Casson $\operatorname{parameter}(\beta)$, Stretching index parameter $(n)$ and velocity slip parameter $(V s)$. Effects of $\gamma^{*}, \gamma, \operatorname{Pr}, S c, K c, \beta$, and $n$ on the steady boundary layers in fluid flow region are discussed in detail. The numerical computation is done using the Matlab in-built numerical solver bvp4c. In the computation we have taken $\eta_{\infty}$, and axis according to the clear figurevisibility. 


\section{RESULT AND ANALYSIS}

In order to validate the method used in this study and to judge the accuracy of the present analysis, comparisons with available results of Andersson [4] ,Mahdy [16] and Ahmed[17] ,corresponding to the skin-friction coefficient $f^{\prime \prime}(0)$ when $n=1$ are presented in Table 1 . As it can be seen, there are excellent agreements between the results. so we are confident that the present numerical method works very efficiently.
For drawing from figures 2 to 40 and from tables 3 to 20 following common parameter values are considered:

$M=1 ; K p=1 ; \quad F s=1 ; \alpha=0.2 ; \beta=0.5 ; n=1 ; \quad V s=0.2$; $\gamma^{*}=0.5 ; \gamma=0.5 ; \operatorname{Pr}=1 ; \quad S c=1 ; K c=0.5 ; \quad S=0.2 ; \lambda t$ $=1 ; \lambda c=1$;

$\mathrm{fO}(3)=0.2 ; \mathrm{fO}(5)=0.2 ; \mathrm{fO}(7)=0.2$;

Here $\mathrm{fO}(3)=f^{\prime \prime}(0), \mathrm{fO}(5)=\theta^{\prime \prime}(0), \quad \mathrm{fO}(7)=$ $\phi^{\prime}(0)$

Table 1 Comparison of $f^{\prime \prime}(0)$ for various values of $V s=[0.0 ; 0.1 ; 0.5 ; 1.0]$ with $\eta_{\infty}=12, \quad \beta=$ inf, $M=0, K p=$ inf, $F s=0$ $n=1, \quad \gamma^{*}=0.0, \quad \gamma=0.0, \alpha=0.0, \quad \operatorname{Pr}=0, \quad S c=0, K c=0.0, \quad S=0.0, \quad \lambda t=0, \quad \lambda c=0, \quad \mathrm{fO}(3)=0.0, \mathrm{f0}(5)=0.0, \mathrm{fO}(7)=0.0$,

\begin{tabular}{|l|l|l|l|l|}
\hline$V s$ & $f^{\prime \prime}(0)$ Andersson [4] & $f^{\prime \prime}(0)$ Mahdy [16] & $\begin{array}{l}f^{\prime \prime}(0) \\
\text { Ahmed[17] }\end{array}$ & $f^{\prime \prime}(0)$ Present study \\
\hline 0.0 & -1.0000 & -1.000000 & -1.0000 & -1.000001136721379 \\
\hline 0.1 & -0.8721 & -0.8721091 & -0.87208 & -0.872083949541335 \\
\hline 0.5 & -0.5912 & -0.591199 & -0.591195 & -0.591197864801179 \\
\hline 1.0 & -0.4302 & -0.4302001 & -0.430160 & -0.430163666996962 \\
\hline
\end{tabular}

Table 2 Data for values of parameters for figures 2 to 11 .

\begin{tabular}{|l|l|l|l|l|l|l|l|}
\hline $\begin{array}{l}\text { Paraeters } \\
\downarrow\end{array}$ & Figure 2 & Figure 3 & Figure 4 & Figure 5,6 & Figure 7,8 & Figure 9,10 & Figure 11 \\
\hline$\gamma^{*}$ & $0.5,1,2,3$ & 0.5 & 0.5 & 0.5 & 0.5 & 0.5 & 0.5 \\
\hline$\gamma$ & 0.5 & $0.5,1,2,3$ & 0.5 & 0.5 & 0.5 & 0.5 & 0.5 \\
\hline $\operatorname{Pr}$ & 1 & 1 & $0.5,1,2,3$ & 1 & 1 & 1 & 1 \\
\hline$S c$ & 1 & 1 & 1 & $0.5,1,2,3$ & 1 & 1 & 1 \\
\hline$K c$ & 0.5 & 0.5 & 0.5 & 0.5 & $0.5,1,2,3$ & 0.5 & 0.5 \\
\hline$\beta$ & 0.5 & 0.5 & 0.5 & 0.5 & 0.5 & $0.5,1,2,3$ & 0.5 \\
\hline$n$ & 1 & 1 & 1 & 1 & 1 & 1 & $0.5,1,2,3$ \\
\hline
\end{tabular}

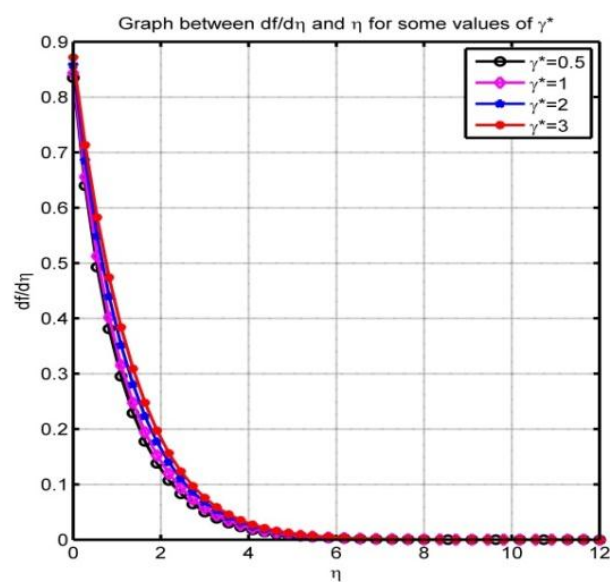

Figure 2 Graph between $f^{\prime}(\eta)$ and $\eta$ for different values of $\gamma^{*}$.

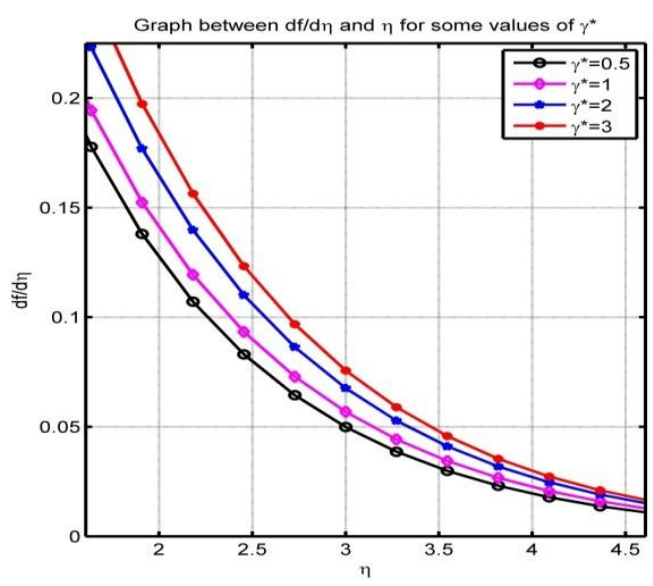

Figure 3 Graph between $f^{\prime}(\eta)$ and $\eta$ for different values of $\gamma^{*}$. 


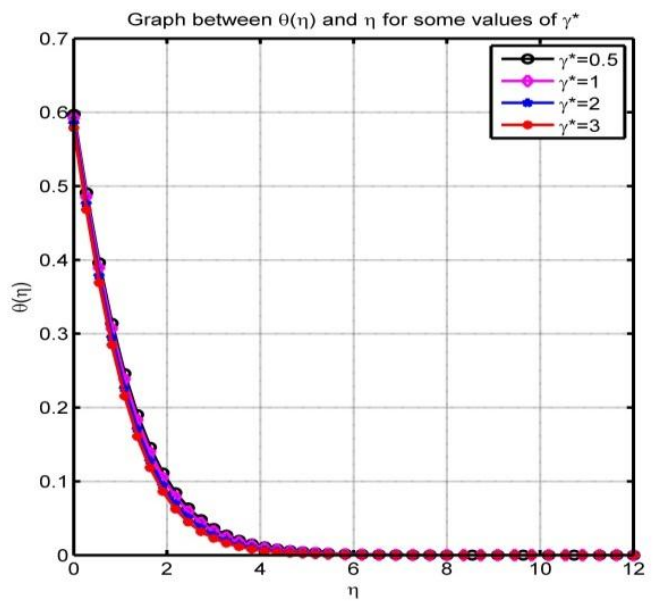

Figure 4 Graph between $\theta(\eta)$ and $\eta$ for different values of $\gamma^{*}$.

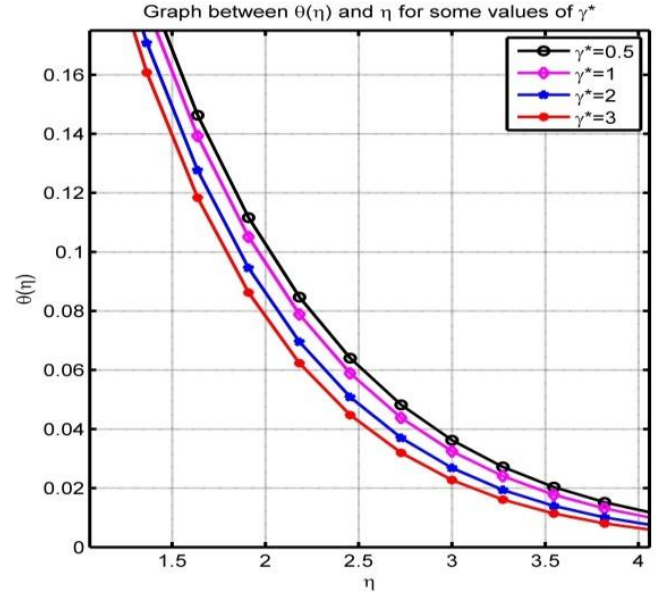

Figure 5 Graph between $\theta(\eta)$ and $\eta$ for different values of $\gamma^{*}$.

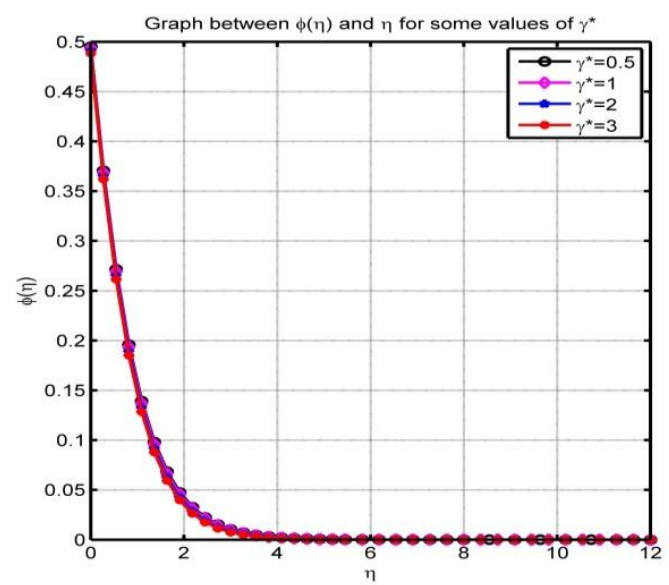

Figure 6 Graph between $\phi(\eta)$ and $\eta$ for different values of $\gamma^{*}$.

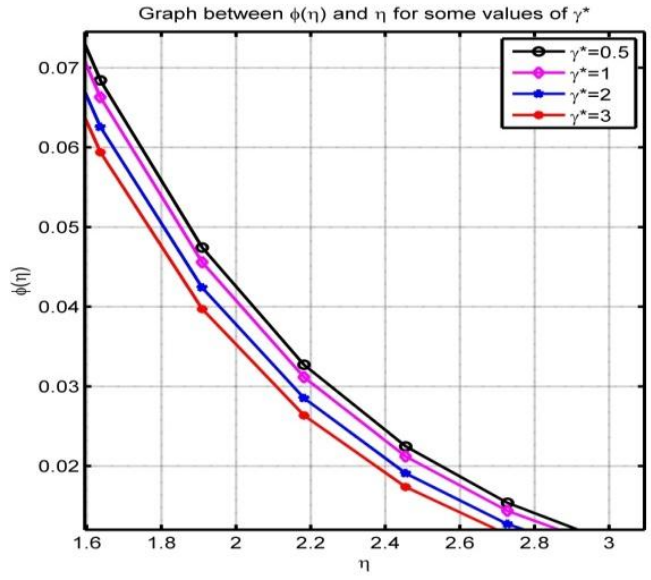

Figure 7 Graph between $\phi(\eta)$ and $\eta$ for different values of $\gamma^{*}$.

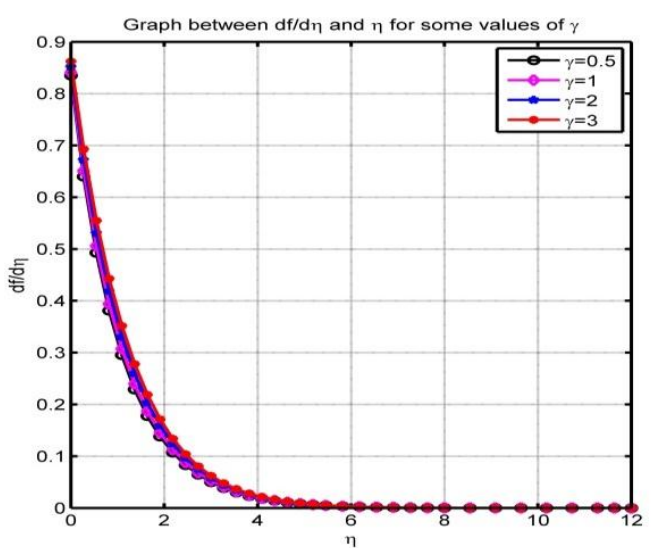

Figure 8 Graph between $f^{\prime}(\eta)$ and $\eta$ for different values of $\gamma$.

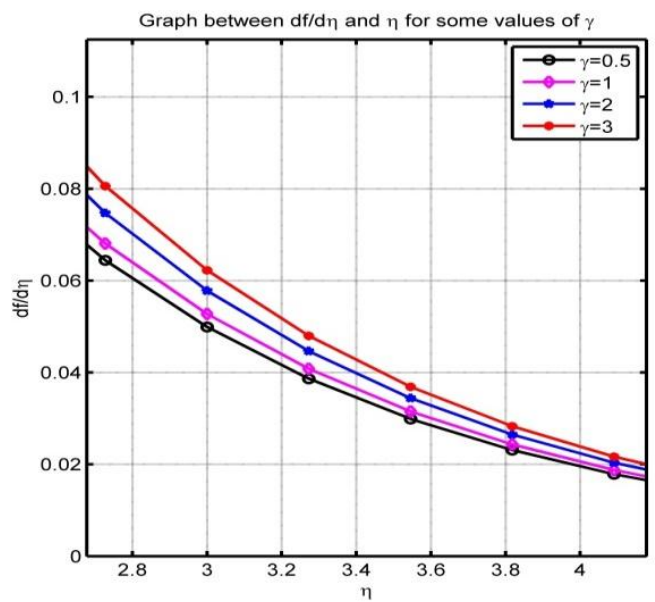

Figure 9 Graph between $f^{\prime}(\eta)$ and $\eta$ for different values of $\gamma$. 


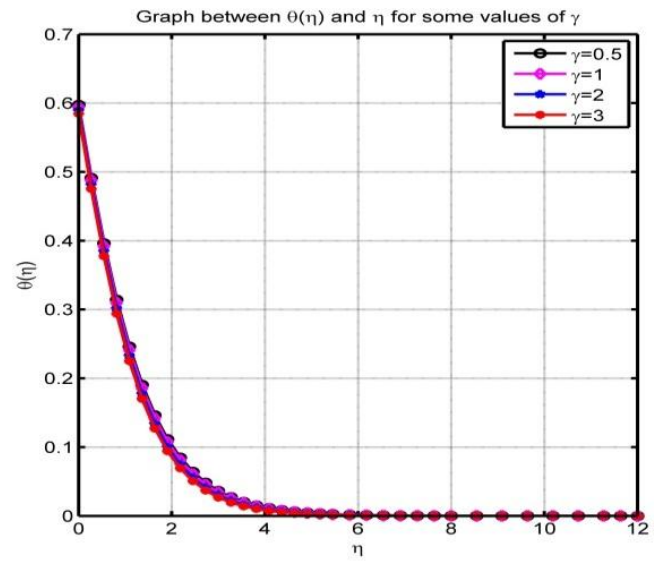

Figure 10 Graph between $\theta(\eta)$ and $\eta$ for different values of $\gamma$.

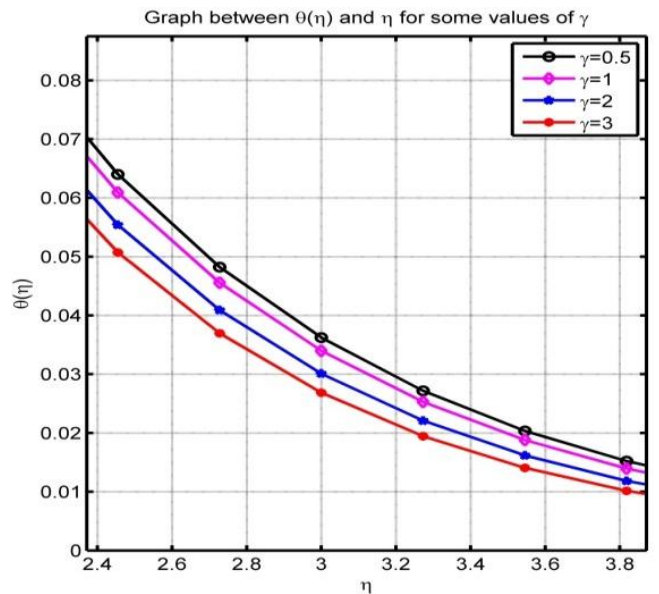

Figure 11 Graph between $\theta(\eta)$ and $\eta$ for different values of $\gamma$.

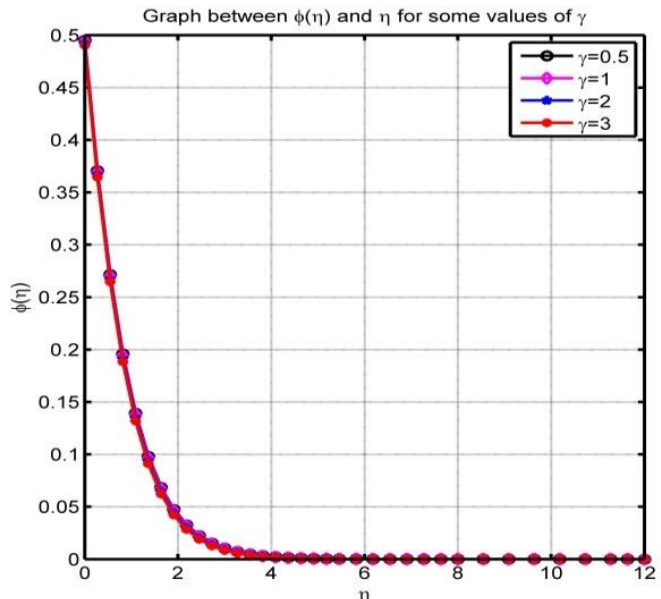

Figure 12 Graph between $\phi(\eta)$ and $\eta$ for different values of $\gamma$.

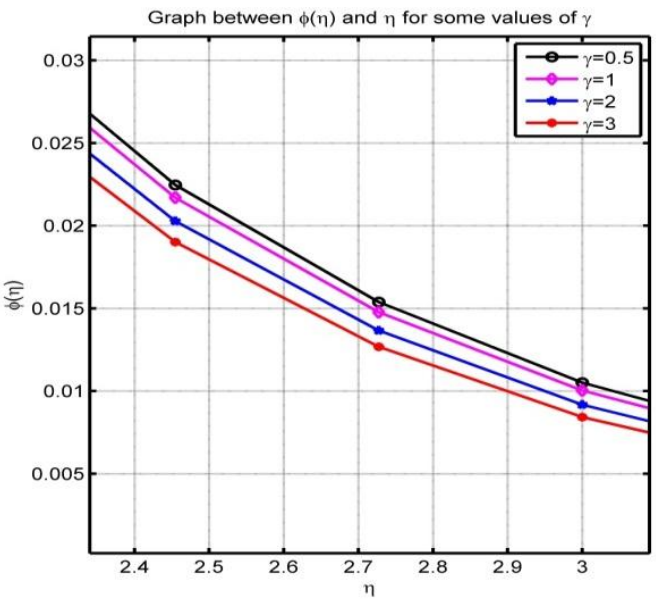

Figure 13 Graph between $\phi(\eta)$ and $\eta$ for different values of $\gamma$.

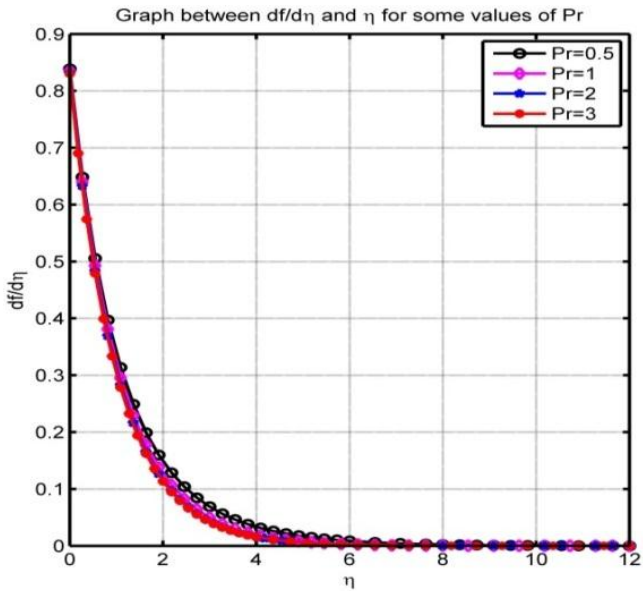

Figure 14 Graph between $f^{\prime}(\eta)$ and $\eta$ for different values of $\mathrm{Pr}$

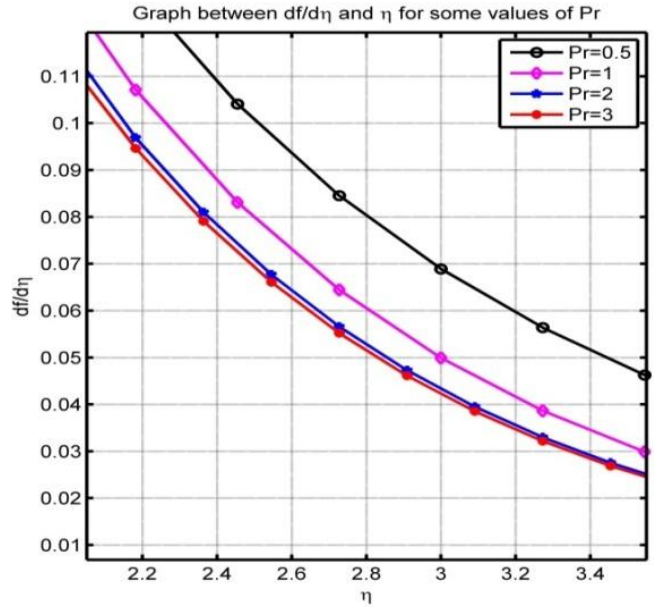

Figure 15 Graph between $f^{\prime}(\eta)$ and $\eta$ for different values of $\operatorname{Pr}$ 


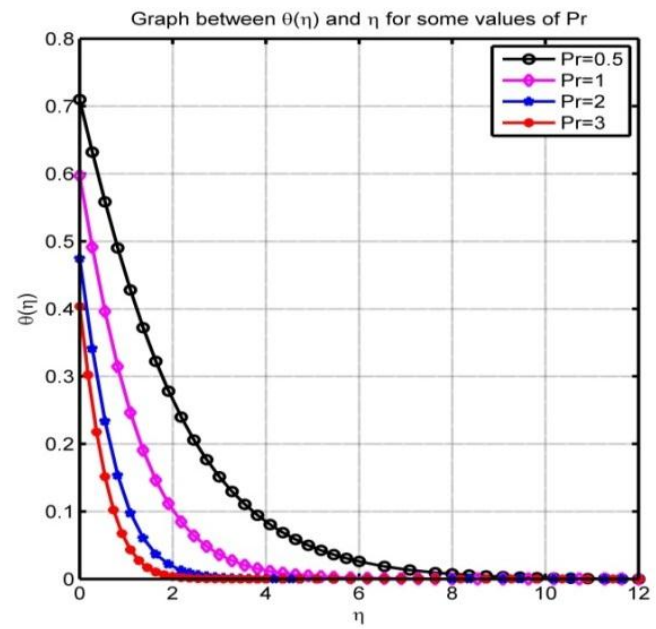

Figure 16 Graph between $\theta(\eta)$ and $\eta$ for different values of $\mathrm{Pr}$

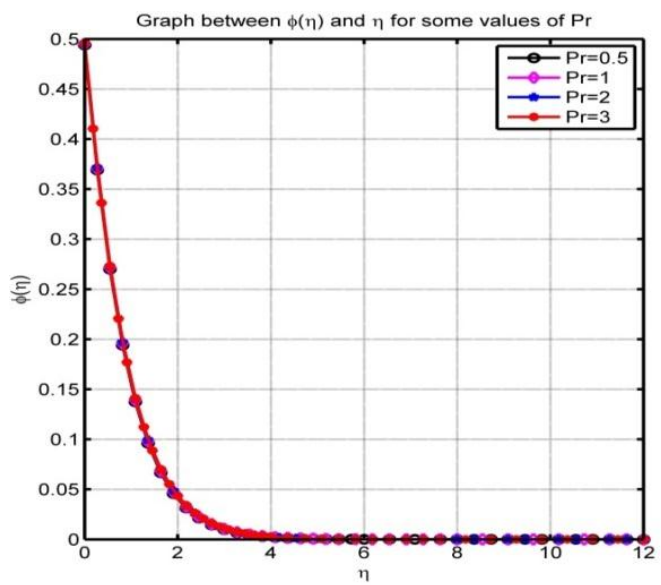

Figure 17 Graph between $\phi(\eta)$ and $\eta$ for different values of $\mathrm{Pr}$

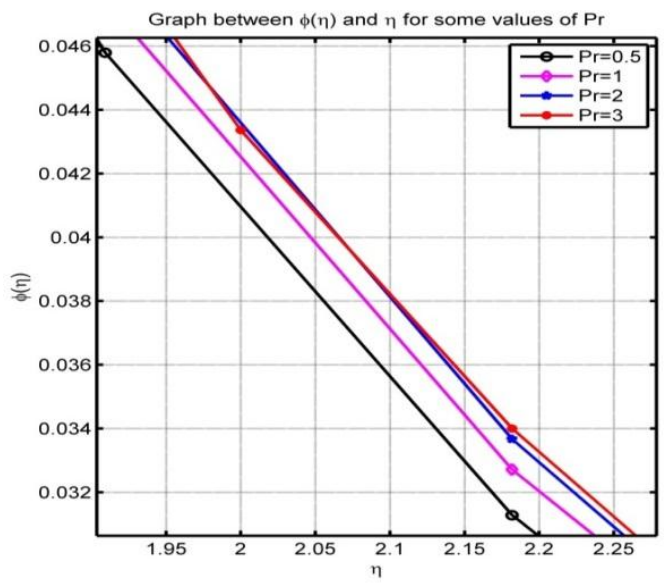

Figure 18 Graph between $\phi(\eta)$ and $\eta$ for different values of $\mathrm{Pr}$

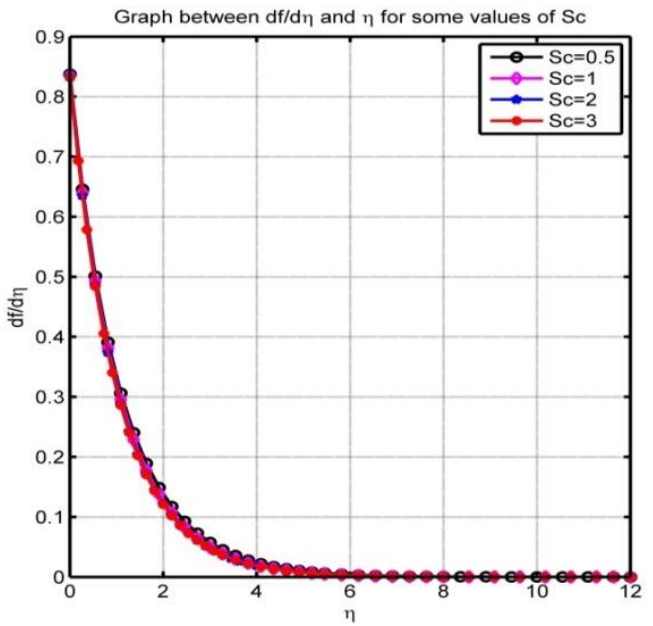

Figure 19 Graph between $f^{\prime}(\eta)$ and $\eta$ for different values of $S c$

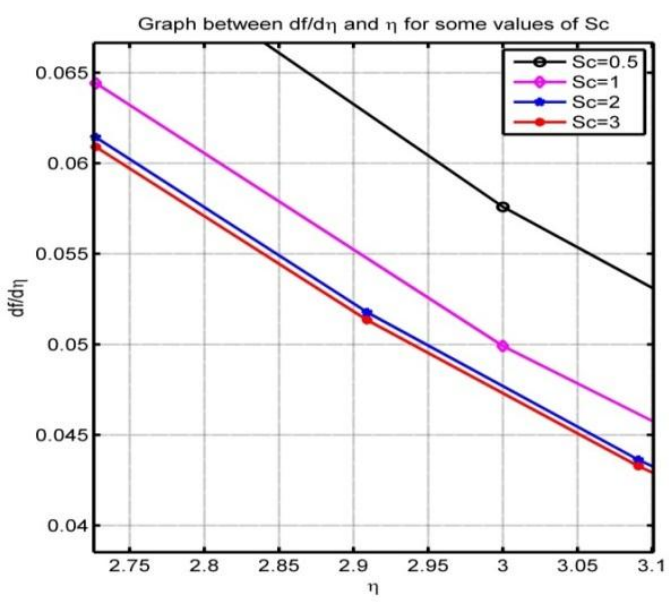

Figure 20 Graph between $f^{\prime}(\eta)$ and $\eta$ for different values of $S c$

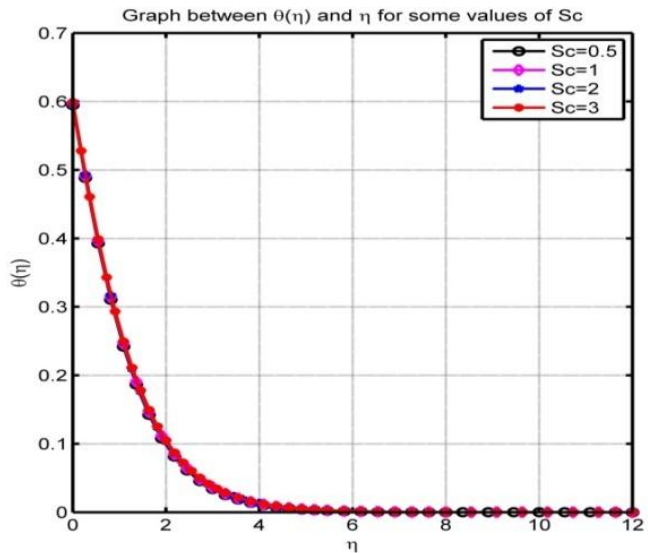

Figure 21 Graph between $\theta(\eta)$ and $\eta$ for different values of $S c$ 


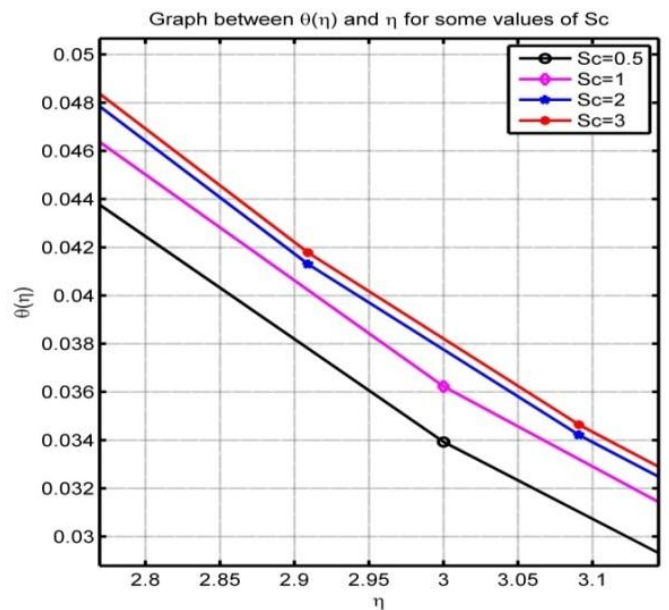

Figure 22 Graph between $\theta(\eta)$ and $\eta$ for different values of $S c$

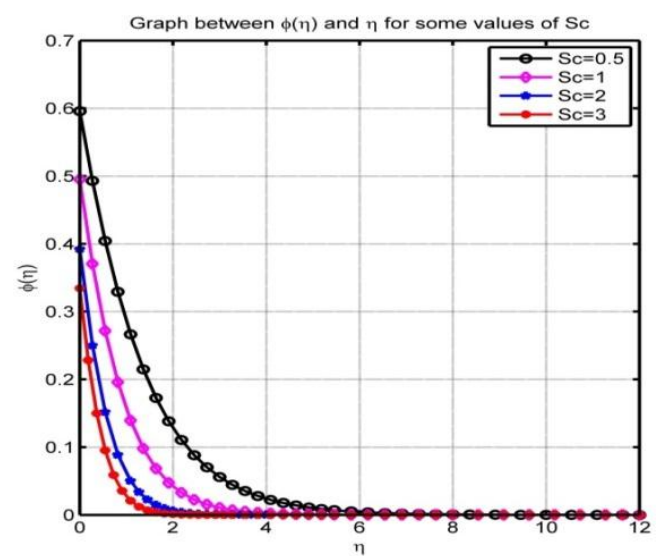

Figure 23 Graph between $\phi(\eta)$ and $\eta$ for different values of $S c$

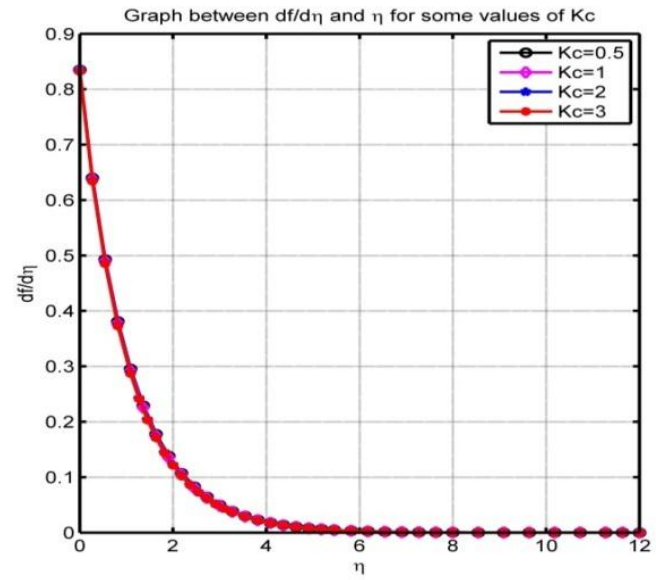

Figure 24 Graph between $f^{\prime}(\eta)$ and $\eta$ for different values of $K c$

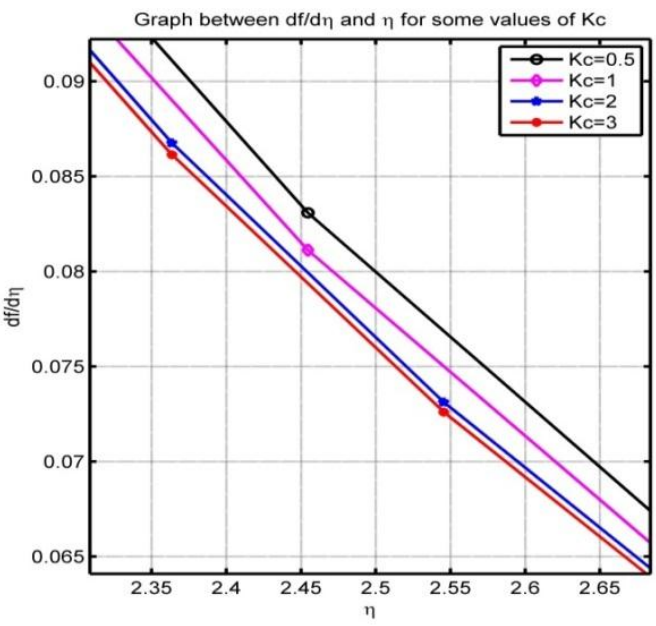

Figure 25 Graph between $f^{\prime}(\eta)$ and $\eta$ for different values of $K c$

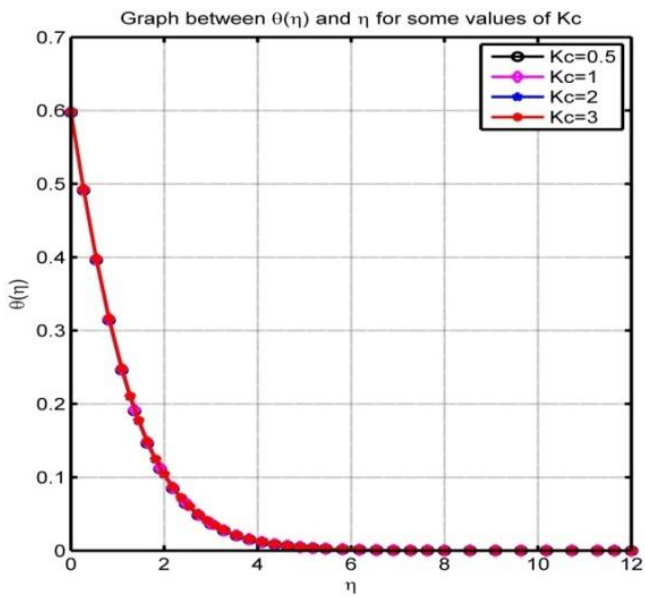

Figure 26 Graph between $\theta(\eta)$ and $\eta$ for different values of $K c$

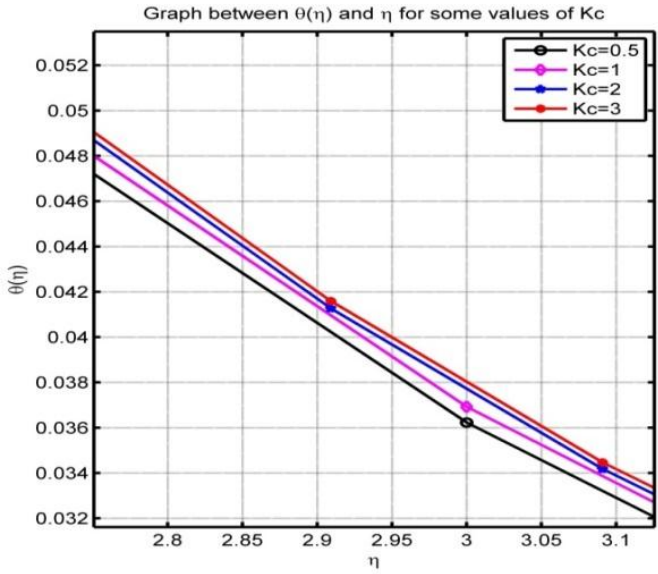

Figure 27 Graph between $\theta(\eta)$ and $\eta$ for different values of $K c$ 


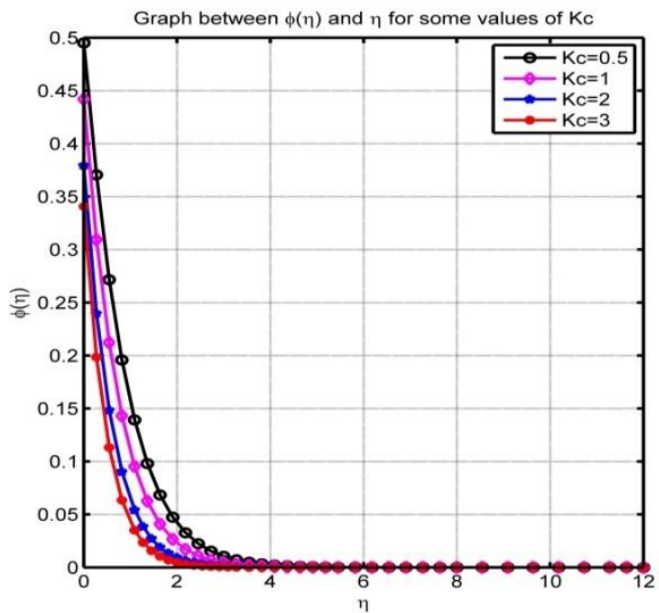

Figure 28 Graph between $\phi(\eta)$ and $\eta$ for different values of $K c$

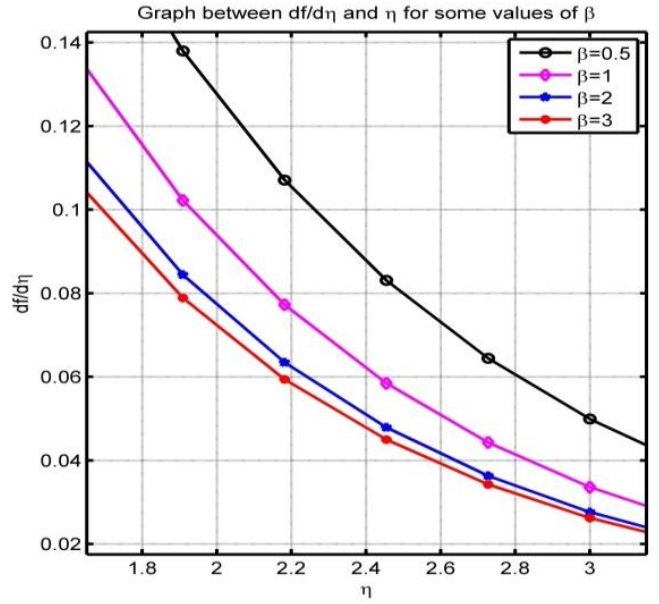

Figure 29 Graph between $f^{\prime}(\eta)$ and $\eta$ for different values of $\beta$.

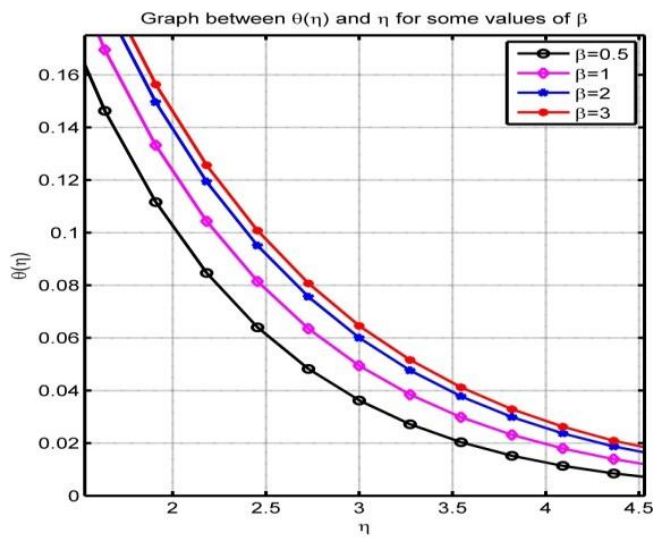

Figure 30 Graph between $\theta(\eta)$ and $\eta$ for different values of $\beta$.

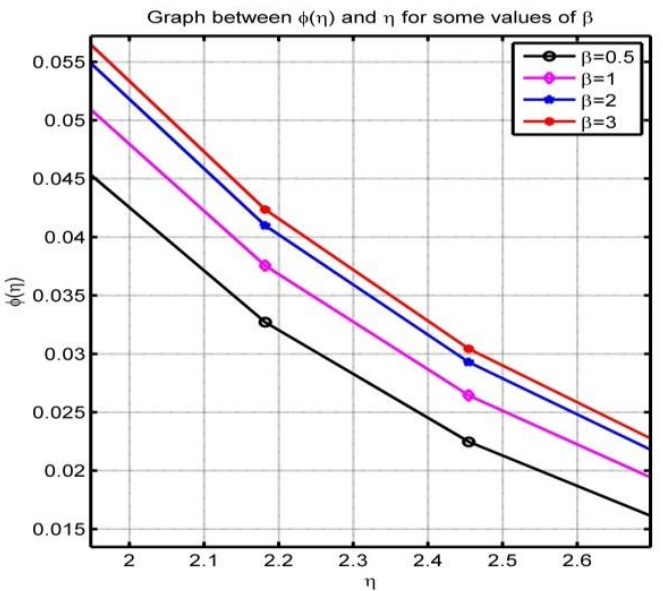

Figure 31 Graph between $\phi(\eta)$ and $\eta$ for different values of $\beta$.

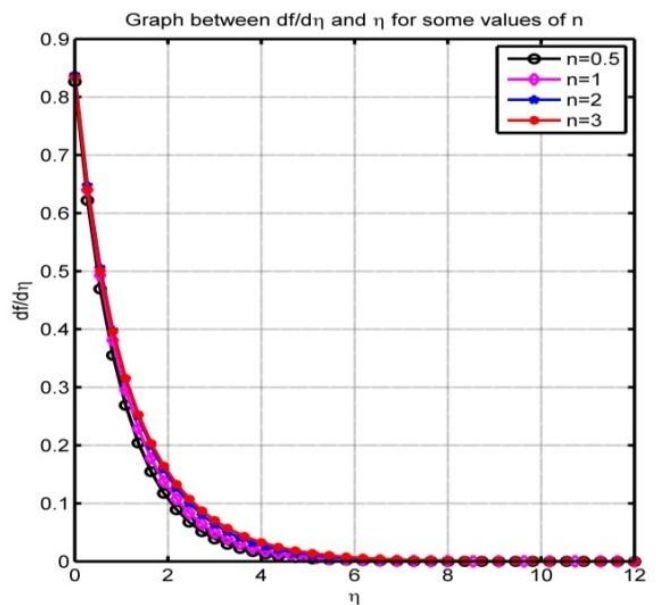

Figure 32 Graph between $f^{\prime}(\eta)$ and $\eta$ for different values of $n$.

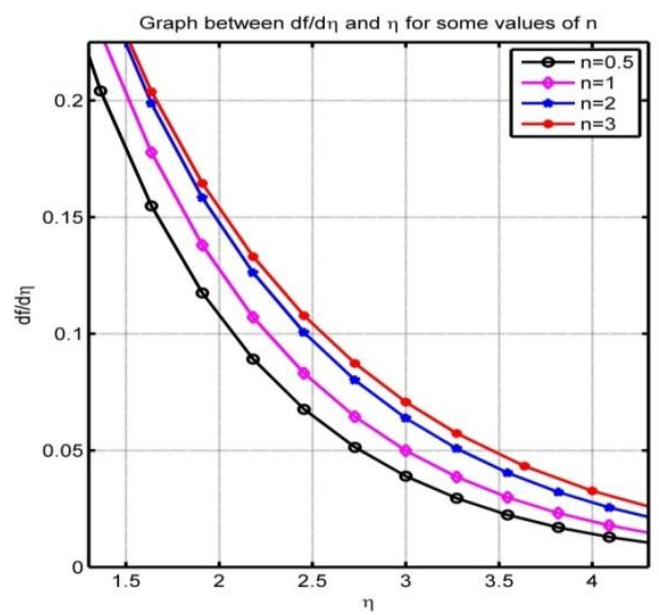

Figure 33 Graph between $f^{\prime}(\eta)$ and $\eta$ for different values of $n$. 


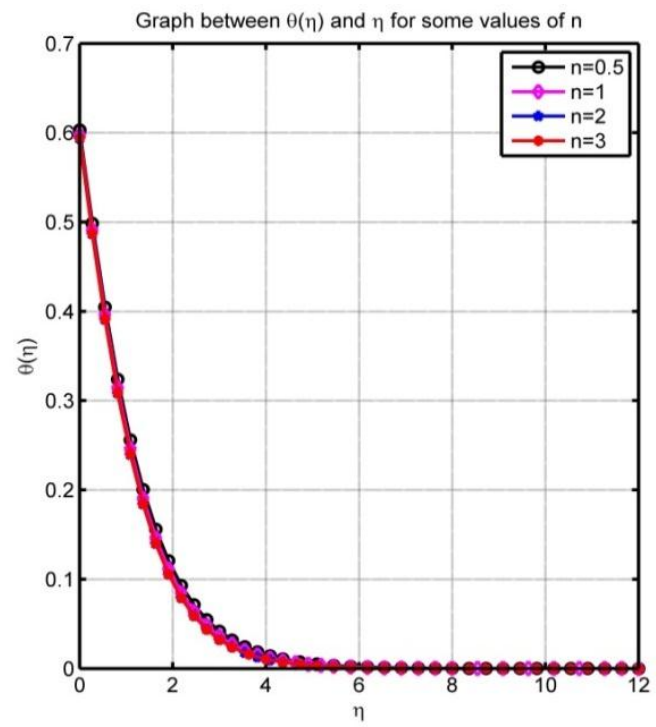

Figure 34 Graph between $\theta(\eta)$ and $\eta$ for different values of $n$.

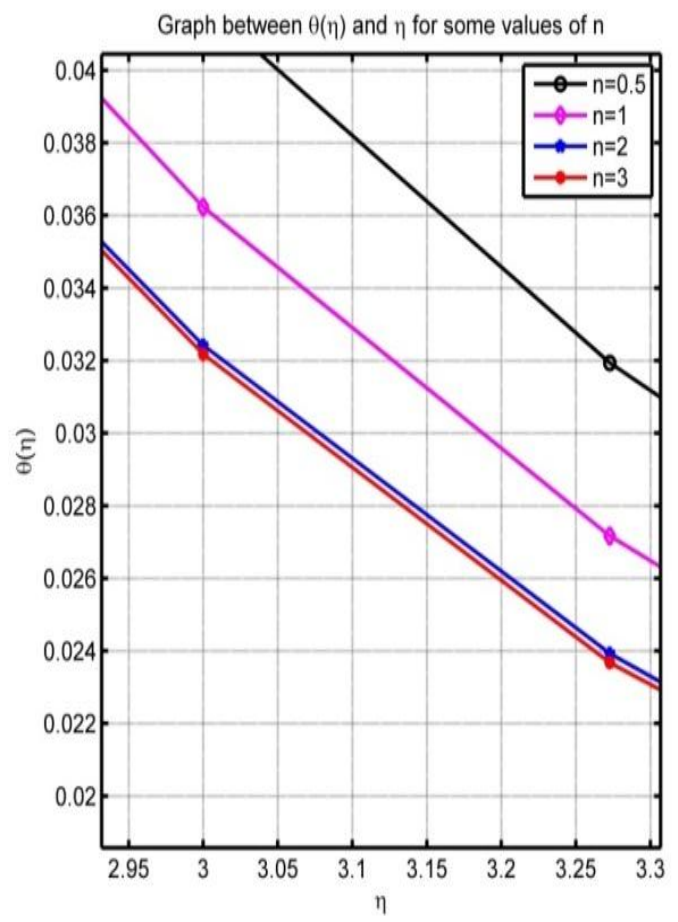

Figure 35 Graph between $\theta(\eta)$ and $\eta$ for different values of $n$.

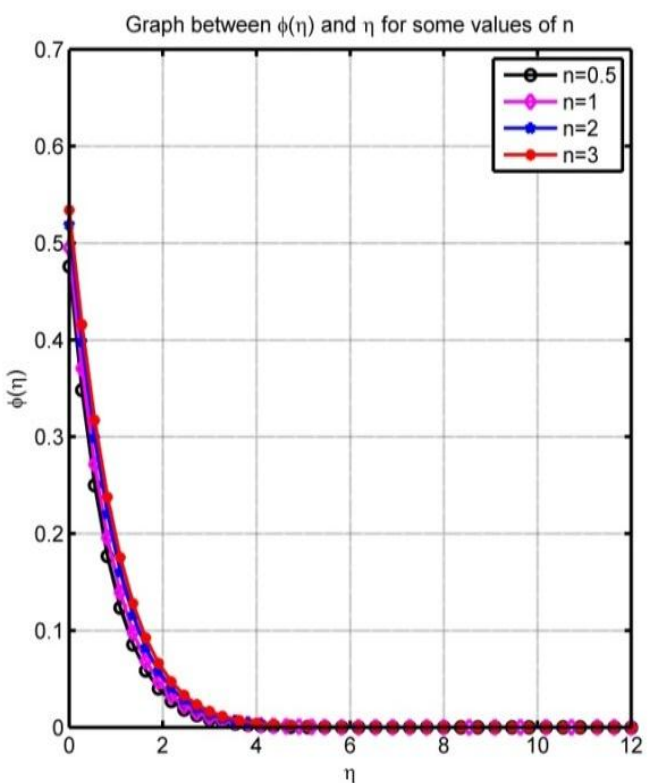

Figure 36 Graph between $\phi(\eta)$ and $\eta$ for different values of $n$

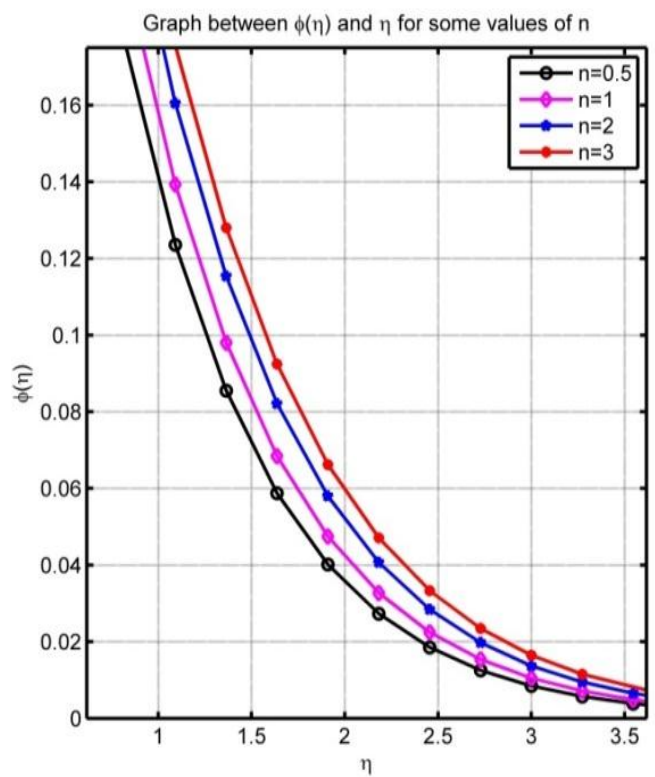

Figure 37 Graph between $\phi(\eta)$ and $\eta$ for different values of $n$ 


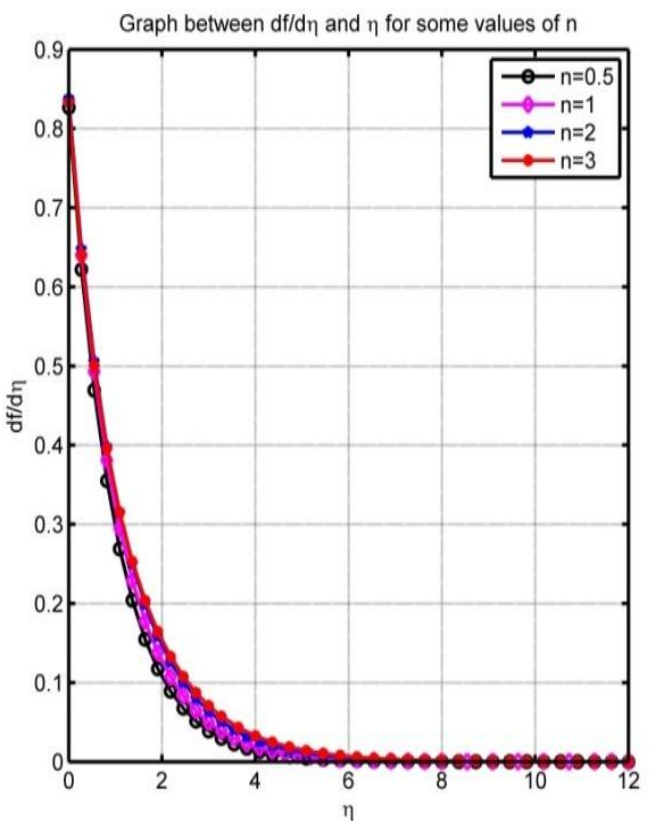

Figure 38 Graph between $f^{\prime}(\eta)$ and $\eta$ for different values of $n$

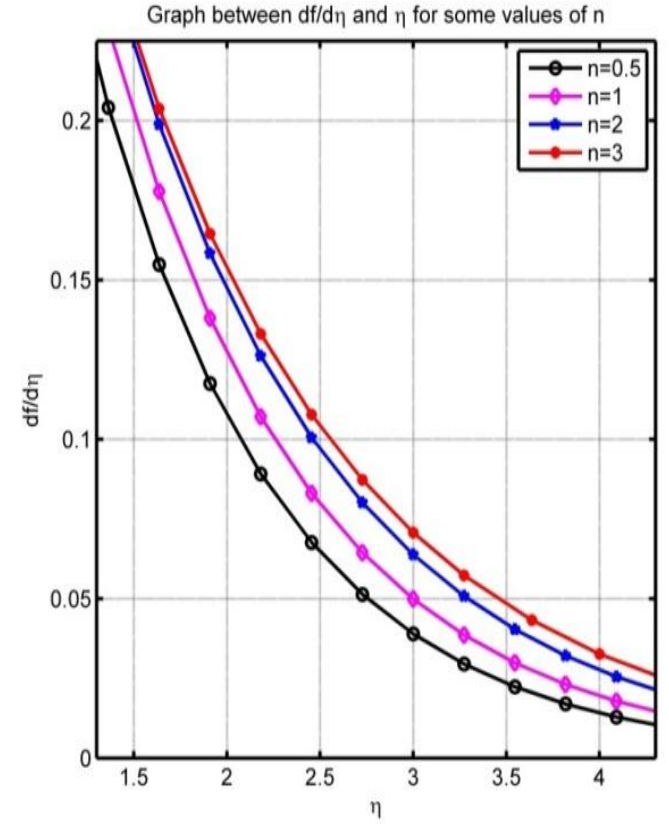

Figure 39 Graph between $f^{\prime}(\eta)$ and $\eta$ for different values of $n$.

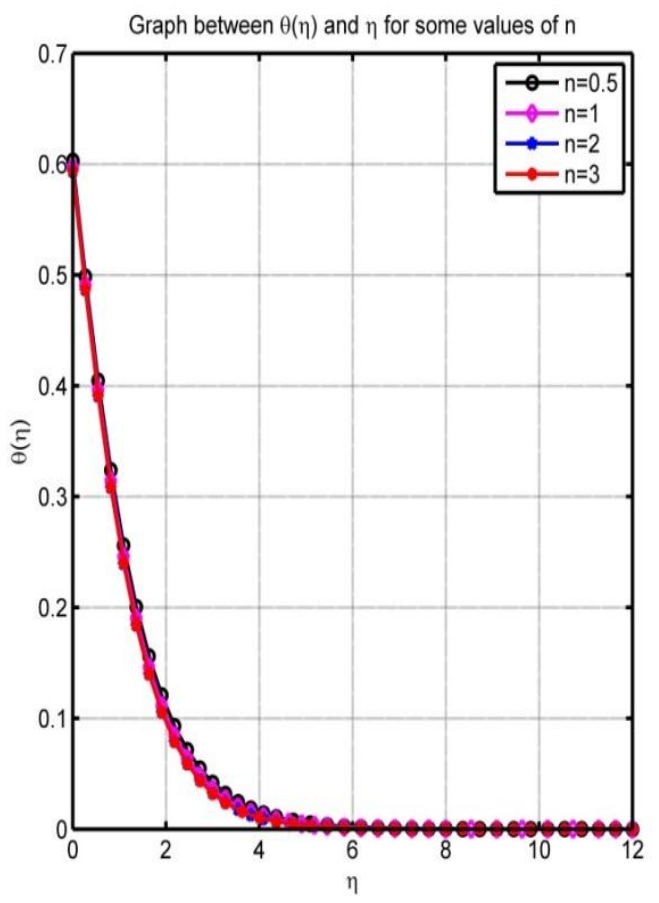

Figure 40 Graph between $\theta(\eta)$ and $\eta$ for different values of $n$.

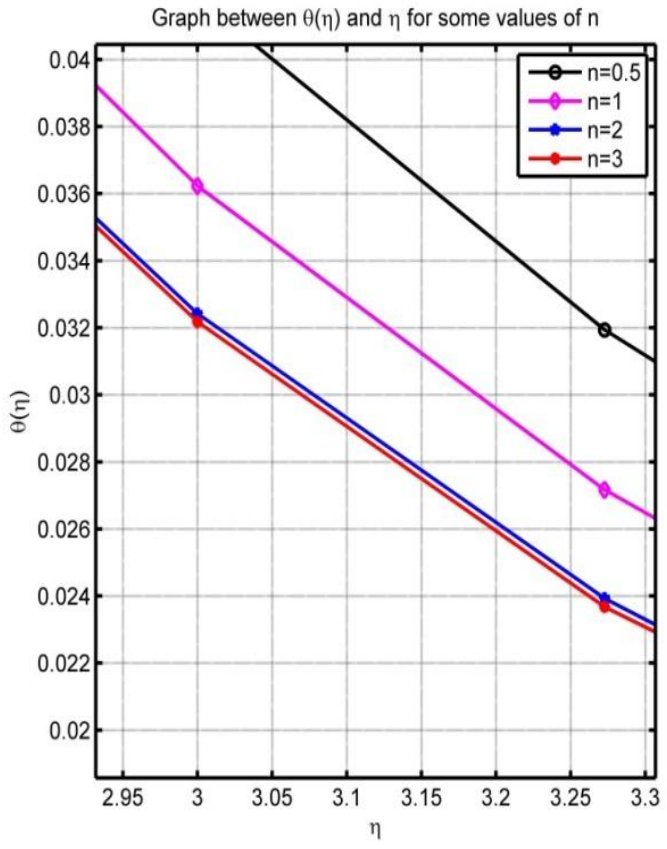

Figure 41 Graph between $\theta(\eta)$ and $\eta$ for different values of $n$. 


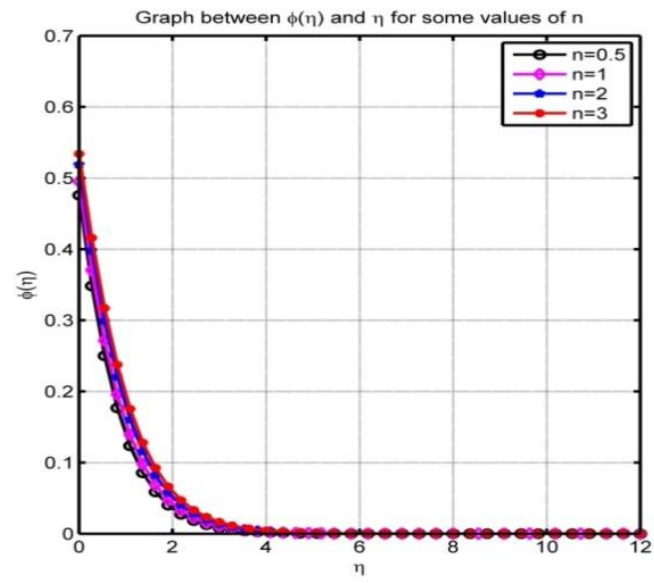

Figure 42 Graph between $\phi(\eta)$ and $\eta$ for different values of $n$

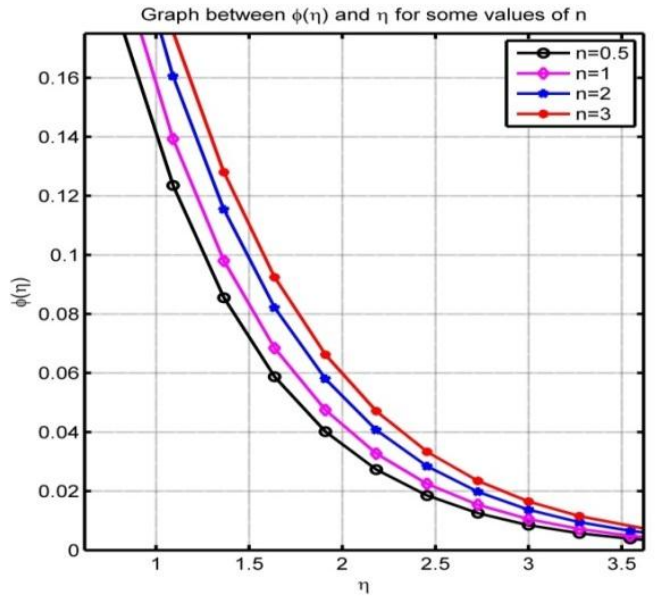

Figure 43 Graph between $\phi(\eta)$ and $\eta$ for different values of $n$.

Figure 3 for $1.5 \leq \eta \leq 4.2$ is part of Figure 2 for $0 \leq \eta \leq 12$. Figure 3 shows velocity boundary layer thickness and the magnitude of the velocity increase and this increase is constant as the Grashof parameter $\gamma^{*}$ increases.

Figure 5 for $1 \leq \eta \leq 4$ is part of Figure 4 for $0 \leq \eta \leq 12$. Figure 5 shows thermal boundary layer thickness and the magnitude of the temperature decrease and this decrease speeds up as the Grashof parameter $\gamma^{*}$ increases.

Figure 7 for $1.6 \leq \eta \leq 3.1$ is part of Figure 6 for $0 \leq \eta \leq 12$. Figure 7 shows concentration boundary layer thickness and the magnitude of the concentration decrease and this decrease speeds up as the Grashof parameter $\gamma^{*}$ increases.
Figure 9 for $2.6 \leq \eta \leq 4.2$ is part of Figure 8 for $0 \leq \eta \leq 12$. Figure 9 shows velocity boundary layer thickness and the magnitude of the velocity increase and this increase speeds up as the modified Grashof parameter $\gamma$ increases.

Figure 11 for $2.4 \leq \eta \leq 3.8$ is part of Figure 10 for $0 \leq \eta \leq 12$. Figure 11 shows thermal boundary layer thickness and the magnitude of the temperature decrease and this decrease speeds up as the modified Grashof parameter $\gamma$ increases.

Figure 13 for $2.4 \leq \eta \leq 3.1$ is part of Figure 12 for $0 \leq \eta \leq 12$. Figure 13 shows concentration boundary layer thickness and the magnitude of the concentration decrease and this decrease speeds up as the modified Grashof parameter $\gamma$ increases.

Figure 15 for $2.1 \leq \eta \leq 3.6$ is part of Figure for $0 \leq \eta \leq 12$. Figure 15 show velocity boundary layer thickness and the magnitude of the velocity decrease and this decrease slows down as the Prandtl number increases.

Figure 16 for $0 \leq \eta \leq 12$. Figure 16 show thermal boundary layer thickness and the magnitude of the temperature decrease and this decrease slows down as the Prandtl number increases.

Figure 18 for $1.9 \leq \eta \leq 2.25$ is part of Figure 17 for $0 \leq \eta \leq 12$. Figure 18 show concentration boundary layer thickness and the magnitude of the concentration increase and this increase slows down as the Prandtl number increases.

Figure 20 for $2.75 \leq \eta \leq 3.1$ is part of Figure 19 for $0 \leq \eta \leq 12$. Figure 20 show velocity boundary layer thickness and the magnitude of the velocity decrease and this decrease slows down as the Schmidth number increases.

Figure 22 for $2.75 \leq \eta \leq 3.15$ is part of Figure 21 for $0 \leq \eta \leq 12$. Figure 22 show thermal boundary layer thickness and the magnitude of the temperature increase and this increase slows down as the Schmidth number increases.

Figure 23 is for $0 \leq \eta \leq 12$. Figure 23 show concentration boundary layer thickness and the 
magnitude of the concentration decrease and this decrease slows down as the Schmidth number increases.

Figure 25 for $2.3 \leq \eta \leq 2.7$ is part of Figure 24 for $0 \leq \eta \leq 12$.

Figure 25 show velocity boundary layer thickness and the magnitude of the velocity decrease and this decrease slows down as the Chemical reaction parameter increases.

Figure 27 for $2.75 \leq \eta \leq 3.15$ is part of Figure 26 for $0 \leq \eta \leq 12$. Figure 27 show thermal boundary layer thickness and the magnitude of the temperature increase and this increase slows down as the Chemical reaction parameter increases.

Figure 28 is for $0 \leq \eta \leq 12$. Figure 28 show concentration boundary layer thickness and the magnitude of the concentration decrease and this decrease slows down as the Chemical reaction parameter increases.

Figure 29 for $1.6 \leq \eta \leq 3.2$ is part of Figure for $0 \leq \eta \leq 12$. Figure 29 shows velocity boundary layer thickness and the magnitude of the velocity decrease and this decrease slows down as the Casson parameter $\beta$ increases.

Figure 30 for $1.5 \leq \eta \leq 4.5$ is part of Figure for $0 \leq \eta \leq 12$. Figure 30 shows thermal boundary layer thickness and the magnitude of the temperature increase and this increase slows down as the Casson parameter $\beta$ increases.

Figure 31 for $2 \leq \eta \leq 3$ is part of Figure for $0 \leq \eta \leq 12$. Figure 31 show concentration boundary layer thickness and the magnitude of the concentration increase and this increase slows down as the Casson parameter $\beta$ increases.

Figure 33 for $1.5 \leq \eta \leq 5$ is part of Figure for $0 \leq \eta \leq 12$. Figure 33 show velocity boundary layer thickness and the magnitude of the velocity increase and this increase slows down as the stretching index parameter $n$ increases.

Figure 35 for $2 \leq \eta \leq 5$ is part of Figure for $0 \leq \eta \leq 12$. Figure 35 ( which is part of Figure 34 ) shows thermal boundary layer thickness and the magnitude of the temperature decrease and this decrease slows down as the stretching index parameter $n$ increases. Transition from $n=2$ to $n=3$ shows wavy nature of the temperature layer at or near the surface.

Figure 37 for $0 \leq \eta \leq 4.5$ is part of Figure for $0 \leq \eta \leq 12$. Figure 37 show concentration boundary layer thickness and the magnitude of the concentration increase and this increase shows no variation as the stretching index parameter $n$ increases.

Figure 39 for $2 \leq \eta \leq 5$ is part of Figure 38 for $0 \leq \eta \leq 12$. Figure 39 show velocity boundary layer thickness and the magnitude of the velocity increase and this increase slows down as the stretching index parameter $n$ increases.

Figure 41 for $2 \leq \eta \leq 5$ is part of Figure for $0 \leq \eta \leq 12$. Figure 41( which is part of Figure 40) shows thermal boundary layer thickness and the magnitude of the temperature decrease and this decrease slows down as the stretching index parameter $n$ increases. Transition from $n=2$ to $n=3$ shows wavy nature of the temperature layer at or near the surface.

Figure 43 for $0 \leq \eta \leq 4.5$ is part of Figure for $0 \leq \eta \leq 12$. Figure 43 show concentration boundary layer thickness and the magnitude of the concentration increase and this increase shows no variation as the stretching index parameter $n$ increases. 
Table 3 Local skin friction $f^{\prime \prime}(0)$ with respect to variation in $\beta$ and $\gamma^{*}$.

\begin{tabular}{|l|l|l|l|l|}
\hline$\gamma^{*} \downarrow \backslash \beta \rightarrow$ & $f^{\prime \prime}(0) \beta=0.5$ & $f^{\prime \prime}(0), \beta=1.0$ & $f^{\prime \prime}(0), \beta=2.0$ & $f^{\prime \prime}(0), \beta=3.0$ \\
\hline 0.5 & -0.8252 & -0.9579 & -1.0604 & -1.1043 \\
\hline 1.0 & -0.7857 & -0.9049 & -0.9960 & -1.0348 \\
\hline 2.0 & -0.7112 & -0.8064 & -0.8777 & -0.9079 \\
\hline 3.0 & -0.6411 & -0.7151 & -0.7692 & -0.7917 \\
\hline
\end{tabular}

Table 4 Local Nusselt Number $-\theta^{\prime}(0)$ with respect to variation in $\beta$ and $\gamma^{*}$.

\begin{tabular}{|l|l|l|l|l|}
\hline$\gamma^{*} \downarrow \backslash \beta \rightarrow$ & $-\theta^{\prime}(0) \beta=0.5$ & $-\theta^{\prime}(0), \beta=1.0$ & $-\theta^{\prime}(0), \beta=2.0$ & $-\theta^{\prime}(0), \beta=3.0$ \\
\hline 0.5 & 0.4028 & 0.3885 & 0.3779 & 0.3736 \\
\hline 1.0 & 0.4073 & 0.3952 & 0.3865 & 0.3830 \\
\hline 2.0 & 0.4149 & 0.4058 & 0.3996 & 0.3972 \\
\hline 3.0 & 0.4213 & 0.4143 & 0.4097 & 0.4079 \\
\hline
\end{tabular}

Table 5 Local Sherwood Number $-\phi^{\prime}(0)$ with respect to variation in $\beta$ and $\gamma^{*}$.

\begin{tabular}{|l|l|l|l|l|}
\hline$\gamma^{*} \downarrow \backslash \beta \rightarrow$ & $-\phi^{\prime}(0) \beta=0.5$ & $-\phi^{\prime}(0), \beta=1.0$ & $-\phi^{\prime}(0), \beta=2.0$ & $-\phi^{\prime}(0), \beta=3.0$ \\
\hline 0.5 & 0.5049 & 0.5002 & 0.4969 & 0.4956 \\
\hline 1.0 & 0.5065 & 0.5023 & 0.4994 & 0.4983 \\
\hline 2.0 & 0.5092 & 0.5059 & 0.5037 & 0.5028 \\
\hline 3.0 & 0.5117 & 0.5090 & 0.5073 & 0.5066 \\
\hline
\end{tabular}

Table 6 Local skin friction $f^{\prime \prime}(0)$ with respect to variation in $\beta$ and $\gamma$.

\begin{tabular}{|l|l|l|l|l|}
\hline$\gamma \downarrow \backslash \beta \rightarrow$ & $f^{\prime \prime}(0) \beta=0.5$ & $f^{\prime \prime}(0), \beta=1.0$ & $f^{\prime \prime}(0), \beta=2.0$ & $f^{\prime \prime}(0), \beta=3.0$ \\
\hline 0.5 & -0.8252 & -0.9579 & -1.0604 & -1.1043 \\
\hline 1.0 & -0.7972 & -0.9207 & -1.0153 & -1.0557 \\
\hline 2.0 & -0.7424 & -0.8482 & -0.9279 & -0.9616 \\
\hline 3.0 & -0.6891 & -0.7779 & -0.8436 & -0.8711 \\
\hline
\end{tabular}

Table 7 Local Nusselt Number $-\theta^{\prime}(0)$ with respect to variation in $\beta$ and $\gamma$.

\begin{tabular}{|l|l|l|l|l|}
\hline$\gamma \downarrow \backslash \beta \rightarrow$ & $-\theta^{\prime}(0) \beta=0.5$ & $-\theta^{\prime}(0), \beta=1.0$ & $-\theta^{\prime}(0), \beta=2.0$ & $-\theta^{\prime}(0), \beta=3.0$ \\
\hline 0.5 & 0.4028 & 0.3885 & 0.3779 & 0.3736 \\
\hline 1.0 & 0.4057 & 0.3925 & 0.3830 & 0.3792 \\
\hline 2.0 & 0.4109 & 0.3998 & 0.3921 & 0.3890 \\
\hline 3.0 & 0.4156 & 0.4063 & 0.3999 & 0.3974 \\
\hline
\end{tabular}

Table 8 Local Sherwood Number $-\phi^{\prime}(0)$ with respect to variation in $\beta$ and $\gamma$.

\begin{tabular}{|l|l|l|l|l|}
\hline$\gamma \downarrow \backslash \beta \rightarrow$ & $-\phi^{\prime}(0) \beta=0.5$ & $-\phi^{\prime}(0), \beta=1.0$ & $-\phi^{\prime}(0), \beta=2.0$ & $-\phi^{\prime}(0), \beta=3.0$ \\
\hline 0.5 & 0.5049 & 0.5002 & 0.4969 & 0.4956 \\
\hline 1.0 & 0.5059 & 0.5015 & 0.4985 & 0.4972 \\
\hline 2.0 & 0.5078 & 0.5040 & 0.5014 & 0.5004 \\
\hline 3.0 & 0.5096 & 0.5063 & 0.5041 & 0.5032 \\
\hline
\end{tabular}


Table 9 Local skin friction $f^{\prime \prime}(0)$ with respect to variation in $\beta$ and $\operatorname{Pr}$.

\begin{tabular}{|l|l|l|l|l|}
\hline $\operatorname{Pr} \downarrow \beta \rightarrow$ & $f^{\prime \prime}(0) \beta=0.5$ & $f^{\prime \prime}(0), \beta=1.0$ & $f^{\prime \prime}(0), \beta=2.0$ & $f^{\prime \prime}(0), \beta=3.0$ \\
\hline 0.5 & -0.8056 & -0.9343 & -1.0340 & -1.0768 \\
\hline 1.0 & -0.8252 & -0.9579 & -1.0604 & -1.1043 \\
\hline 2.0 & -0.8416 & -0.9794 & -1.0858 & -1.1314 \\
\hline 3.0 & -0.8488 & -0.9894 & -1.0981 & -1.1447 \\
\hline
\end{tabular}

Table 10 Local Nusselt Number $-\theta^{\prime}(0)$ with respect to variation in $\beta$ and $\operatorname{Pr}$.

\begin{tabular}{|l|l|l|l|l|}
\hline $\operatorname{Pr} \downarrow \backslash \beta \rightarrow$ & $-\theta^{\prime}(0) \beta=0.5$ & $-\theta^{\prime}(0), \beta=1.0$ & $-\theta^{\prime}(0), \beta=2.0$ & $-\theta^{\prime}(0), \beta=3.0$ \\
\hline 0.5 & 0.2902 & 0.2767 & 0.2676 & 0.2641 \\
\hline 1.0 & 0.4028 & 0.3885 & 0.3779 & 0.3736 \\
\hline 2.0 & 0.5255 & 0.5139 & 0.5048 & 0.5009 \\
\hline 3.0 & 0.5964 & 0.5871 & 0.5796 & 0.5764 \\
\hline
\end{tabular}

Table 11 Local Sherwood Number with respect to variation in $\beta$ and $\operatorname{Pr}$.

\begin{tabular}{|l|l|l|l|l|}
\hline $\operatorname{Pr} \downarrow \backslash \beta \rightarrow$ & $-\phi^{\prime}(0) \beta=0.5$ & $-\phi^{\prime}(0), \beta=1.0$ & $-\phi^{\prime}(0), \beta=2.0$ & $-\phi^{\prime}(0), \beta=3.0$ \\
\hline 0.5 & 0.5060 & 0.5016 & 0.4985 & 0.4972 \\
\hline 1.0 & 0.5049 & 0.5002 & 0.4969 & 0.4956 \\
\hline 2.0 & 0.5041 & 0.4991 & 0.4956 & 0.4941 \\
\hline 3.0 & 0.5038 & 0.4987 & 0.4950 & 0.4935 \\
\hline
\end{tabular}

Table 12 Local skin friction $f^{\prime \prime}(0)$ with respect to variation in $\beta$ and $S c$.

\begin{tabular}{|l|l|l|l|l|}
\hline$S c \downarrow \backslash \beta \rightarrow$ & $f^{\prime \prime}(0) \beta=0.5$ & $f^{\prime \prime}(0), \beta=1.0$ & $f^{\prime \prime}(0), \beta=2.0$ & $f^{\prime \prime}(0), \beta=3.0$ \\
\hline 0.5 & -0.8114 & -0.9410 & -1.0412 & -1.0841 \\
\hline 1.0 & -0.8252 & -0.9579 & -1.0604 & -1.1043 \\
\hline 2.0 & -0.8362 & -0.9721 & -1.0770 & -1.1219 \\
\hline 3.0 & -0.8411 & -0.9786 & -1.0848 & -1.1304 \\
\hline
\end{tabular}

Table 13 Local Nusselt Number $-\theta^{\prime}(0)$ with respect to variation in $\beta$ and $S c$.

\begin{tabular}{|l|l|l|l|l|}
\hline$S c \downarrow \backslash \beta \rightarrow$ & $-\theta^{\prime}(0) \beta=0.5$ & $-\theta^{\prime}(0), \beta=1.0$ & $-\theta^{\prime}(0), \beta=2.0$ & $-\theta^{\prime}(0), \beta=3.0$ \\
\hline 0.5 & 0.4052 & 0.3918 & 0.3820 & 0.3780 \\
\hline 1.0 & 0.4028 & 0.3885 & 0.3779 & 0.3736 \\
\hline 2.0 & 0.4013 & 0.3863 & 0.3752 & 0.3706 \\
\hline 3.0 & 0.4008 & 0.3855 & 0.3741 & 0.3694 \\
\hline
\end{tabular}

Table 14 Local Sherwood Number $-\phi^{\prime}(0)$ with respect to variation in $\beta$ and $S c$.

\begin{tabular}{|l|l|l|l|l|}
\hline$S c \downarrow \backslash \beta \rightarrow$ & $-\phi^{\prime}(0) \beta=0.5$ & $-\phi^{\prime}(0), \beta=1.0$ & $-\phi^{\prime}(0), \beta=2.0$ & $-\phi^{\prime}(0), \beta=3.0$ \\
\hline 0.5 & 0.4040 & 0.3995 & 0.3964 & 0.3952 \\
\hline 1.0 & 0.5049 & 0.5002 & 0.4969 & 0.4956 \\
\hline 2.0 & 0.6077 & 0.6037 & 0.6007 & 0.5994 \\
\hline 3.0 & 0.6655 & 0.6620 & 0.6594 & 0.6583 \\
\hline
\end{tabular}


Table 15 Local skin friction $f^{\prime \prime}(0)$ with respect to variation in $\beta$ and $K c$.

\begin{tabular}{|l|l|l|l|l|}
\hline$K c \downarrow \backslash \beta \rightarrow$ & $f^{\prime \prime}(0) \beta=0.5$ & $f^{\prime \prime}(0), \beta=1.0$ & $f^{\prime \prime}(0), \beta=2.0$ & $f^{\prime \prime}(0), \beta=3.0$ \\
\hline 0.5 & -0.8252 & -0.9579 & -1.0604 & -1.1043 \\
\hline 1.0 & -0.8308 & -0.9653 & -1.0693 & -1.1139 \\
\hline 2.0 & -0.8366 & -0.9730 & -1.0785 & -1.1238 \\
\hline 3.0 & -0.8398 & -0.9772 & -1.0836 & -1.1292 \\
\hline
\end{tabular}

Table 16 Local Nusselt Number $-\theta^{\prime}(0)$ with respect to variation in $\beta$ and $K c$.

\begin{tabular}{|l|l|l|l|l|}
\hline$K c \downarrow \backslash \beta \rightarrow$ & $-\theta^{\prime}(0) \beta=0.5$ & $-\theta^{\prime}(0), \beta=1.0$ & $-\theta^{\prime}(0), \beta=2.0$ & $-\theta^{\prime}(0), \beta=3.0$ \\
\hline 0.5 & 0.4028 & 0.3885 & 0.3779 & 0.3736 \\
\hline 1.0 & 0.4021 & 0.3873 & 0.3764 & 0.3719 \\
\hline 2.0 & 0.4014 & 0.3863 & 0.3750 & 0.3704 \\
\hline 3.0 & 0.4010 & 0.3857 & 0.3744 & 0.3697 \\
\hline
\end{tabular}

Table 17 Local Sherwood Number $-\phi^{\prime}(0)$ with respect to variation in $\beta$ and $K c$.

\begin{tabular}{|l|l|l|l|l|}
\hline$K c \downarrow \backslash \beta \rightarrow$ & $-\phi^{\prime}(0) \beta=0.5$ & $-\phi^{\prime}(0), \beta=1.0$ & $-\phi^{\prime}(0), \beta=2.0$ & $-\phi^{\prime}(0), \beta=3.0$ \\
\hline 0.5 & 0.5049 & 0.5002 & 0.4969 & 0.4956 \\
\hline 1.0 & 0.5582 & 0.5555 & 0.5536 & 0.5528 \\
\hline 2.0 & 0.6208 & 0.6194 & 0.6184 & 0.6180 \\
\hline 3.0 & 0.6593 & 0.6584 & 0.6578 & 0.6576 \\
\hline
\end{tabular}

Table 18 Local skin friction $f^{\prime \prime}(0)$ with respect to variation in $\beta$ and $n$.

\begin{tabular}{|l|l|l|l|l|}
\hline$n \downarrow \backslash \beta \rightarrow$ & $f^{\prime \prime}(0) \beta=0.5$ & $f^{\prime \prime}(0), \beta=1.0$ & $f^{\prime \prime}(0), \beta=2.0$ & $f^{\prime \prime}(0), \beta=3.0$ \\
\hline 0.5 & -0.8673 & -1.0079 & -1.1166 & -1.1632 \\
\hline 1.0 & -0.8252 & -0.9579 & -1.0604 & -1.1043 \\
\hline 2.0 & -0.8111 & -0.9393 & -1.0377 & -1.0798 \\
\hline 3.0 & -0.8331 & -0.9625 & -1.0616 & -1.1039 \\
\hline
\end{tabular}

Table 19 Local Nusselt Number $-\theta^{\prime}(0)$ with respect to variation in $\beta$ and $n$.

\begin{tabular}{|l|l|l|l|l|}
\hline$n \downarrow \backslash \beta \rightarrow$ & $-\theta^{\prime}(0) \beta=0.5$ & $-\theta^{\prime}(0), \beta=1.0$ & $-\theta^{\prime}(0), \beta=2.0$ & $-\theta^{\prime}(0), \beta=3.0$ \\
\hline 0.5 & 0.3970 & 0.3814 & 0.3699 & 0.3653 \\
\hline 1.0 & 0.4028 & 0.3885 & 0.3779 & 0.3736 \\
\hline 2.0 & 0.4066 & 0.3934 & 0.3838 & 0.3799 \\
\hline 3.0 & 0.4061 & 0.3934 & 0.3842 & 0.3805 \\
\hline
\end{tabular}

Table 20 Local Sherwood Number $-\phi^{\prime}(0)$ with respect to variation in $\beta$ and $n$.

\begin{tabular}{|l|l|l|l|l|}
\hline$n \downarrow \backslash \beta \rightarrow$ & $-\phi^{\prime}(0) \beta=0.5$ & $-\phi^{\prime}(0), \beta=1.0$ & $-\phi^{\prime}(0), \beta=2.0$ & $-\phi^{\prime}(0), \beta=3.0$ \\
\hline 0.5 & 0.5242 & 0.5202 & 0.5174 & 0.5163 \\
\hline 1.0 & 0.5049 & 0.5002 & 0.4969 & 0.4956 \\
\hline 2.0 & 0.4811 & 0.4753 & 0.4712 & 0.4696 \\
\hline 3.0 & 0.4660 & 0.4594 & 0.4547 & 0.4529 \\
\hline
\end{tabular}


Table 3 shows for fix value of the Grashop parameter $\gamma *$ as the Casson parameter $\beta$ increases, local skin friction $f^{\prime \prime}(0)$ decreases and for fix value of the Casson parameter $\beta$ as the Grashop parameter $\gamma^{*}$ increases skin friction $f^{\prime \prime}(0)$ increases.

Table 4 shows for fix value of the Grashop number $\gamma^{*}$, as the Casson parameter $\beta$ increases, Local Nusselt Number $-\theta^{\prime}(0)$ decreases and for fix value of the Casson parameter $\beta$ as the Grashop number $\gamma^{*}$ increases Local Nusselt Number $-\theta^{\prime}(0)$ increases.

Table 5 shows for fix value of the Grashop number $\gamma^{*}$, as the Casson parameter $\beta$ increases, Local Sherwood Number $-\phi^{\prime}(0)$ decreases and for fix value of the Casson parameter $\beta$ as the Grashop number $\gamma^{*}$ increases Local Sherwood Number $-\phi^{\prime}(0)$ increases.

Table 6 shows for fix value of the modified Grashop parameter $\gamma$, as the Casson parameter $\beta$ increases, local skin friction $f^{\prime \prime}(0)$ decreases and for fix value of the Casson parameter $\beta$ as the modified Grashop parameter $\gamma$ increases skin friction $f^{\prime \prime}(0)$ increases.

Table 7 shows for fix value of the modified Grashop number $\gamma$, as the Casson parameter $\beta$ increases, Local Nusselt Number $-\theta^{\prime}(0)$ decreases and for fix value of the Casson parameter $\beta$ as the modified Grashop number $\gamma$ increases Local Nusselt Number $-\theta^{\prime}(0)$ increases.

Table 8 shows for fix value of the modified Grashop number $\gamma$, as the Casson parameter $\beta$ increases, Local Sherwood Number $-\phi^{\prime}(0)$ decreases and for fix value of the Casson parameter $\beta$ as the modified Grashop number $\gamma$ increases Local Sherwood Number $-\phi^{\prime}(0)$ increases.

Table 9 shows for fix value of the Prandtl number $\operatorname{Pr}$, as the Casson parameter $\beta$ increases, local skin friction $f^{\prime \prime}(0)$ decreases and for fix value of the Casson parameter $\beta$ as the Prandtl number $\operatorname{Pr}$ increases skin friction $f^{\prime \prime}(0)$ decreases.

Table 10 shows for fix value of the Prandtl number $\operatorname{Pr}$, as the Casson parameter $\beta$ increases, Local Nusselt Number $-\theta^{\prime}(0)$ decreases and for fix value of the Casson parameter $\beta$ as the Prandtl number $\operatorname{Pr}$ increases Local Nusselt Number $-\theta^{\prime}(0)$ increases.

Table 11 shows for fix value of the Prandtl number $\operatorname{Pr}$, as the Casson parameter $\beta$ increases, Local Sherwood Number $-\phi^{\prime}(0)$ decreases and for fix value of the Casson parameter $\beta$ as the Prandtl number $\operatorname{Pr}$ increases Local Sherwood Number $-\phi^{\prime}(0)$ decreases.

Table 12 shows for fix value of the Schmidth number $S c$, as the Casson parameter $\beta$ increases, local skin friction $f^{\prime \prime}(0)$ decreases and for fix value of the Casson parameter $\beta$ as the Schmidth number $S c$ increases skin friction $f^{\prime \prime}(0)$ decreases.

Table 13 shows for fix value of the Schmidth number $S c$, as the Casson parameter $\beta$ increases, Local Nusselt Number $-\theta^{\prime}(0)$ decreases and for fix value of the Casson parameter $\beta$ as the Schmidth number $S c$ increases Local Nusselt Number $-\theta^{\prime}(0)$ decreases.

Table 14 shows for fix value of the Schmidth number $S c$, as the Casson parameter $\beta$ increases, Local Sherwood Number $-\phi^{\prime}(0)$ decreases and for fix value of the Casson parameter $\beta$ as the Schmidth number $S c$ increases Local Sherwood Number $-\phi^{\prime}(0)$ increases.

Table 15 shows for fix value of the chemical reaction parameter $K c$, as the Casson parameter $\beta$ increases, local skin friction $f^{\prime \prime}(0)$ decreases and for fix value of the Casson parameter $\beta$ as the chemical reaction parameter $K c$ increases skin friction $f^{\prime \prime}(0)$ decreases.

Table 16 shows for fix value of the chemical reaction parameter $K c$, as the Casson parameter $\beta$ increases, Local Nusselt Number $-\theta^{\prime}(0)$ decreases and for fix value of the Casson parameter $\beta$ as the chemical reaction parameter $K c$ increases Local Nusselt Number $-\theta^{\prime}(0)$ decreases.

Table 17 shows for fix value of the chemical reaction parameter $K c$, as the Casson parameter $\beta$ increases, Local Sherwood Number $-\phi^{\prime}(0)$ decreases and for fix 
value of the Casson parameter $\beta$ as the chemical reaction parameter $K c$ increases Local Sherwood Number $-\phi^{\prime}(0)$ increases.

Table 18 shows for fix value of the stretching index parameter $n$, as the Casson parameter $\beta$ increases, local skin friction $f^{\prime \prime}(0)$ decreases and for fix value of the Casson parameter $\beta$ as the stretching index parameter $n$ increases skin friction $f^{\prime \prime}(0)$ increases.

Table 19 shows for fix value of the stretching index parameter $n$, as the Casson parameter $\beta$ increases, Local Nusselt Number $-\theta^{\prime}(0)$ decreases and for fix value of the Casson parameter $\beta$ as the stretching index parameter, $n$ increases Local Nusselt Number $-\theta^{\prime}(0)$ increases.

Table 20 shows for fix value of the stretching index parameter $n$, as the Casson parameter $\beta$ increases, Local Sherwood Number $-\phi^{\prime}(0)$ decreases and for fix value of the Casson parameter $\beta$ as the stretching index parameter, $\square$ increases Local Sherwood Number $-\phi^{\prime}(0)$ decreases.

\section{CONCLUSION}

In this paper, we have studied numerically the effect of Grashof number, modified Grashof number and chemical reaction on the non-Darcy MHD flow of a Casson fluid over a nonlinearly stretching sheet in a porous medium. In the mathematical model, using similarity variables, the momentum, energy and concentration equations are transformed to non-dimensional ordinary differential equations. These equations are solved numerically using bvp4c method, a Matlab in- built bvp4c-programm. A discussion for the effects of the parameters involved on the boundary layer regions and the magnitude of the velocity, temperature and concentration and local Skin friction, Local Nusselt number and Local Sherwood number have been done graphically and numerically using figures and tables. From this investigation, we have drawn the following conclusions:

From the graphs we have following conclusion:

(i) As the Prandtl number Schmidth number the Chemical reaction parameter, the Casson parameter increases, velocity boundary layer thickness and magnitude of velocity decreases.

(ii) As the Chemical reaction parameter, Schmidth number the Casson parameter increases thermal boundary layer thickness and magnitude of temperature increases.

(iii) As the Prandtl number, the Schmidth number, the Casson parameter, the stretching index parameter increases, concentration boundary layer thickness and magnitude of concentration increases.

(iv) As the Grashof parameter, modified Grashof parameter, the stretching index parameter increases, velocity boundary layer thickness and magnitude of velocity increases.

(v) As the Grashof parameter, modified Grashof parameter, the Prandtl number, the stretching index parameter increases, the thermal jump(thermal slip) parameter thermal boundary layer thickness and magnitude of temperature decreases.

(vi) As the Grashof parameter, modified Grashof parameter, the Schmidth number, the Chemical reaction parameter increases concentration boundary layer thickness and magnitude of concentration decreases.

From the tables we have following conclusion:

(vii) for fix value of for fix value of Grashof number ,modified Grashof number, Prandtl number, Schmidth number, Chemical reaction parameter, or stretching index parameter , as Casson parameter increases Local skin friction or Local Nusselt Number increases or Local Sherwood Number decreases.

(viii) for fix value of Casson parameter , as Grashof number or modified Grashof numberincreases Local skin friction or Local Nusselt Number increases or Local Sherwood Number increases.

(ix) for fix value of Casson parameter, as Prandtl number increases Local skin friction or Local Sherwood Number decreases but Local Nusselt Number increases .

(x) for fix value of Casson parameter, as Schmidth number or Chemical reaction parameter increases Local skin friction or Local Nusselt Number decreases but Local Sherwood Number increases.

(xi) for fix value of Casson parameter, as stretching index parameter increases Local skin friction or Local Sherwood Number increases but Local Nusselt Number decreases.

\section{ACKNOWLEDGEMENT}

Author expresses sincere thanks to the Department of Mathematics, Doon University, Dehradun, India, for its support and cooperation in the work.

\section{COMPETING INTERESTS}

Author has declared that no competing interests exist.

\section{REFERENCES}

[1] Lawrence J Crane. "Flow Past a Stretching Plate", Kurze Mitteilungen, Brief Report- Communications breves, Vol.21;1970.

[2] Rajagopal K R, Na TY, and Gupta A S, "Flow of viscoelastic fluid over a stretching sheet", Rheologica Acta 23:213-215;1984.

[3] Ishak Anuar, Nazar Roslinda, and Pop Ioan, "Falkner-Skan equation for flow past a moving wedge with suction or injection", $\mathbf{J}$. Appl. Math. \& Computing Vol. 25, No. 1 - 2, pp. 67 - 83 ;2007. Website: http://jamc.net.

[4] Siddappa B. and Abel Subhas, "Non-Newtonian flow past a stretching plate", Journal of Applied Mathematics and Physics (ZAMP),Vol.36; November 1985. 
[5] Andersson H L, "Note: MHD flow of a viscoelastic fluid past a stretching surface", Acta Mechanica 95,227-230; 1992.

[6] Dandapat B S, Holmedal L E and Andersson H L, "Note: on the stability of MHD flow of a viscoelastic fluid past a stretching sheet", Acta Mechanica 130,143-146;1998 .

[7] Fang Tiegang, Guo Fang, Lee Chia-fon F, "A note on the extended Blasius equation”, Applied Mathematics Letters 19, 613-617;2006. www.elsevier.com/locate/aml, www.sciencedirect.com.

[8] Mamaloukas Ch, Spartalis S, Manussaridis Z, "Similarity Approach to the Problem of Second Grade Fluid Flows over a Stretching Sheet", Applied Mathematical Sciences, Vol. 1, no. 7, 327 - 338;2007.

[9] Khidir Ahmed, "A note on the solution of general Falkner-Skan problem by two novel semi-analytical techniques", Propulsion and Power Research 4(4):212-220;2015. http://ppr.buaa.edu.cn/,www.sciencedirect.com.

[10] Bataller Rafael Cortell, "Numerical Comparisons of Blasius and Sakiadis Flows", Matematika, Volume 26, Number 2, 187-196; 2010.

[11] Motsa S S, Hayat T, Aldossary O M, "MHD flow of upperconvected Maxwell fluid over porous stretching sheet using successive Taylor series linearization method", Appl. Math. Mech. -Engl. Ed., 33(8), 975-990;2012. DOI 10.1007/s10483-012-1599-x .

[12] Motsa Sandile Sydney, Sibanda Precious, "On the solution of MHD flow over a nonlinear stretching sheet by an efficient semianalytical technique", International Journal for Numerical Methods in Fluids. April 2012, DOI: 10.1002/fld.2541.

[13] Rosca A, "Mhd boundary-layer flow over a permeable shrinking surface”, Acta Universitatis Apulensis ,No. 36, pp. 31-38, ISSN: 1582-5329.(result verified); 2013.

[14] Nadeem S, Hussain S T, and Lee Changhoon, "Flow of a williamson fluid over a stretching sheet" , Brazilian Journal of Chemical Engineering, Vol. 30, No. 03, pp. 619 - 625, July - September, 2013.

[15] Mukhopadhyay Swati, "MHD boundary layer slip flow along a stretching cylinder", Ain Shams Engineering Journal 4, 317324;2013. www. elsevier.com/locate/asej,www.sciencedirect.com.

[16] Akbar N S, Nadeem S, Haq Rizwan Ul, Ye Shiwei, "MHD stagnation point flow of Carreau fluid toward a permeable shrinking sheet: Dual solutions", Ain Shams Engineering Journal 5, 12331239;2014. www .elsevier .com /locate /asej , www.sciencedirect.com.

[17] Nadeem S, Haq Rizwan Ul, Lee C, "Research note: MHD flow of a Casson fluid over an exponentially shrinking sheet", Scientia Iranica B 19 (6), 1550-1553;2012. www.sciencedirect.com.

[18] Biswas R, Mondal M, Sarkar D R and Ahmmed S F, "Effects of radiation and chemical reaction on MHD unsteady heat and mass transfer of Casson fluid flow past a vertical plate", Journal of Advances in Mathematics and Computer Science, Vol. 23(2), 1-16. http://www.science domain.org/issue/2795.

[19] Ahmmed S F, Biswas R, and Afikuzzaman M, "Unsteady MHD free convection flow of nanofluid through an exponentially accelerated inclined plate embedded in a porous medium with variable thermal conductivity in the presence of radiation", Journal of Nanofluids, Vol. 7, pp. 891-901. http://www.aspbs. com/jon.htm.

[20] Biswas Rajib, Ahmmed Sarder Firoz, "Effects of Hall current and chemical reaction on MHD unsteady heat and mass transfer of Casson nanofluid flow through a vertical plate", Journal of Heat Transfer.http://asmedigital collection.asme.org.

[21] Noor Khan Saeed, Zuhra Samina, Shah Zahir, Bonyah Ebenezer, Khan Waris, Saeed Islam, Khan Aurangzeb, "Hall current and thermophoresis effects on magnetohydrodynamic mixed convective heat and mass transfer thin film flow", Journal of Physics Communications ;2018. https://doi.org/10.1088/2399-6528/aaf830.

[22] Sharada K, Shankar B, “MHD Mixed Convection Flow of a casson fluid over an exponentially stretching surface with the effects of soret, dufour, thermal radiation and chemical reaction", World Journal of Mechanics.;5:165-177, 2015. Available:http://dx.doi.org/10.4236/wjm.2015.59017.

[23] Swati Mukhopadhyay, Krishnendu Bhattacharyya, Tasawar Hayat, "Exact solutions for the flow of Casson fluid over a stretching surface with transpiration and heat transfer effects", Chin Phys.;22(11):114701, 2013. DOI: 10.1088/1674-1056/22/11/114701.
[24] N. Pandya,, M. S. Quraishi, "Combined Effect of Variable Permeability and Variable Magnetic field on MHD Flow past an inclined plate with exponential Temperature and Mass Diffusion with Chemical reaction through Porous media" , International Journal of Scientific Research in Physics and Applied Sciences, Vol.6, Issue.5, pp.62-68, October (2018) Research Paper E-ISSN: 2348-3423.

[25] Kala Bhim Sen, "Analysis of Non-Darcy MHD flow of a Casson Fluid over a Non-linearly Stretching Sheet with Partial Slip in a Porous Medium",AJARR, 3(3): 1-15, 2019; Article no.AJARR.47539.

\section{AUTHOR'S PROFILE}

Dr. Bhim Sen Kala, M.Sc, PhD, working in Fluid Mechanics through Numerical Analysis, currently teaching under Department of Mathematics, Doon University, Dehradun, 248001, Uttarakhand, India.Email: bhimskala@gmail.com 